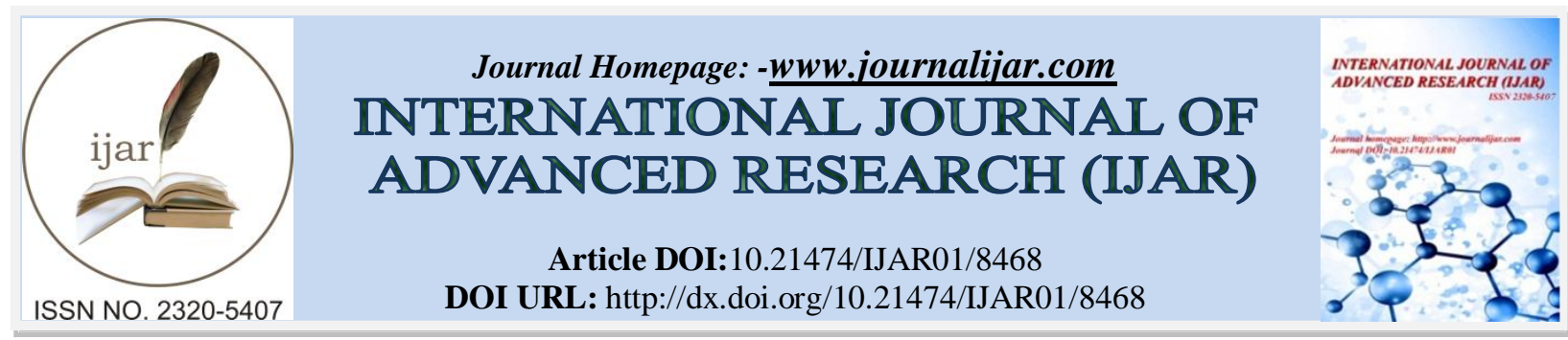

RESEARCH ARTICLE

\title{
NUTRITIONAL PROSPECTS, ANTIOXIDANT AND ANTIBACTERIAL ACTIVITIES OF SOME FOOD PLANTS CONSUMED BY WILD MANDRILL (MANDRILLUS SPHINX) POPULATION FROM LÉKÉDI PARK (BAKOUMBA, GABON).
}

\section{Gontran nsi akoue $^{1,2}$, Pierre Philippe Mbehang Nguema $^{3,4}$, Richard Onanga ${ }^{3}$, Arsene Mabika Mabika ${ }^{3}$ and Ibrahim B. . .}

1. Laboratoire de Physiologie Animale: Electrophysiologie-Pharmacologie-URAB, Université des Sciences et Techniques de Masuku, BP 913, Franceville Gabon.

2. Laboratoire de Sciences de la Vie et de la Terre de l'Ecole Normale Supérieur de Libreville (LaSciViT), BP : 17009Libreville-Gabon.

3. Laboratoire de Bactériologie de l'Unité de Recherche et d'Analyses Médicales du Centre International de Recherche Médicale de Franceville Gabo.

4. Institut de Recherche en Ecologie Tropicale du Centre International de Recherche Scientifique et Technologique de Libreville, Gabon.

\section{Manuscript Info}

Manuscript History

Received: 02 December 2018

Final Accepted: 04 January 2019

Published: February 2019

Key words:-

Mandrill diet, nutritional potential, antioxidant, antibacterial, zoopharmacognosy approach.

\section{Abstract}

Research on wild vegetables established that several vegetables supply significant quantities of essential nutrients to the body and, assist in the maintenance of health and prevention of diseases.

This study consisted to evaluated nutritional and pharmacological potentials of some wild vegetables from Mandrillus sphinx diet. Vegetables species selected according to their consumption frequencies and ethnopharmacological uses by local population, were therefore subjected to nutritional and pharmacological analyses.

Proximate nutrients values showed that fruits and leaves from mandrill diet present the high values in crude protein, fats and carbohydrate.

Results showed also high mineral amounts were also obtained with the majority of plants eaten.

Phytochemical and pharmacological analyses have shown the highest content of secondary compounds had ranged between $0.06 \pm 1.18$ and $44.46 \pm 0.10 \mathrm{~g}$ EAG/100g of dried matter (total phenols compounds amount), Antinutritional compounds amounts varies between $0.005 \pm$ 2.1 and $28.15 \pm 0.22 \mathrm{~g} \mathrm{EAT} / 100 \mathrm{~g}$ DM (tannins) and $0.021 \pm 0.29$ and $11.45 \pm 0.03 \mathrm{~g} \mathrm{EQ} / 100 \mathrm{~g}$ DM (flavonoids).

Our results showed also the good antioxidant activity of some plant organs consumed by both two populations human and mandrill such as Aframomum cf. polyanthum, A. alboviolaceum, Pseudospondias longifolia, Chrysophyllum lacourtianum and Landolphia mannii. The lowest values have recorded with fruits of Antrocaryon klaineanum, Uapaca guineensis and Pentaclethra macrophylla has been also observed.

Among plants analyzed for antibacterial activities, Tristemma mauritianum, Macaranga schweinfurthii, Medelina mirabilis, Alchornea floribunda and Pentaclethra eetveldeana present the 
bactericidal effects. This study showed that vegetables from mandrill diet constitute good nutritional sources of nutrients and mineral compounds. Some plants will be also good natural antioxidants sources and antibacterial usable in human health.

Thus, investigations of mandrill diet will constitute the good sources of nutrients and antinutritional compounds to improvement of nutrition, health and an approach for drugs discovery.

Copy Right, IJAR, 2019,. All rights reserved. 


\section{Introduction:-}

Diseases such as cancer, cardiovascular, Alzheimer's diseases, neurodegenerative disorders, many chronic affections and food security represent a major challenge of public health in the developed countries, where some people and mainly children still suffer from malnutrition including deficiencies in micronutrients due to the lack of affordable and nutritious food. Several epidemiological studies have proved that these metabolic affections were associated with oxidative stress phenomena (Zia-ul-haq et al., 2008).

However, in many low-income countries to fight against the under and malnutrition, several food programs those involve the use of non-timber forest products (NTFPs) in the diet, including wild edible plants, are undertaken. The major contribution of NTFPs is well recognized in the improvement of nutrition and health in urban and rural area in low-income countries (Fernanda et al., 2006).

In Africa, many studies reported that a vast number of indigenous wild plants play a significant role in the diet of the population (Mouhammed et al., 2011).

Also, Food and Agricultural Organization (FAO) reported that about one billion of population use wild food in their diet (Burhingame, 2000). These wild edible plants contain valuable food ingredients which may be successfully used to build up and repair the body. Thus, they would play an important role in human diets supplying the body with low calories and substantial amounts of necessary minerals and vitamins (Fasuyi, 2006) since they provide amounts of proteins, fats, carbohydrates, fibers, vitamins and minerals (Onwordi et al., 2009; Saikia and Deka, 2013) necessary for the good functioning of organism.

They also contain many secondary metabolites with beneficial effects on the human health. It is the case of bioactive molecules such as phenolic compounds which have strong antioxidant effect against free radicals and reactive oxygen species involved like major causes in chronic human diseases such as cardiovascular diseases, diabetes and degenerative diseases (Kyung-Hee et al., 2005; Chen and Yen, 2007).

Ongoing studies in the Lékédi Park (Bakoumba, Gabon) carried out on mandrill (Mandrillus sphinx) diet behavior have shown that many wild plants are consumed by human and non-human primates. Some of them were used by non-human primates only (Nsi Akoué et al., 2017; Nsi Akoué, 2017). Certain reports argue for the underutilization and lack of the nutritional value of wild vegetables around the world (Keatinge, 2012).

In order to contribute to the fighting against food vulnerability accentuated and to prevent some pathologies as bacterial and metabolic affections in rural area, we aimed to the study antioxidant potential, antibacterial activity and nutritional value of wild edible plants consumed by free mandrill population from the Lékédi Park (Bakoumba) which may be very useful in human diet and as medicinal alternative.

\section{Material and methods:- Study site and population}

The study was conducted in the Lékédi Park located $7 \mathrm{~km}$ North-East of the village of Bakoumba in Southern Gabon. The park is composed of 14000 hectares of forests interspersed with savannas and divided into three modules (Nsi Akoué et al., 2017).

The study population of mandrills consisted with habituated population of $\sim 130$ free-ranging individuals living in the Lékédi Park and its vicinity. This study community's home range, covered about an area of 2400 ha (Nsi Akoué et al., 2017). She is originated from 65 captive individuals initially housed at the CIRMF (Centre International de Recherche Médicale de Franceville) and released in 2002 and 2006 (Peignot et al., 2008). At the present time, the group is composed of about $85 \%$ are wild-born individuals (Brockmeyer et al., 2015; Nsi Akoué et al., 2017).

Behavioural data collection

Behavioral data on the feeding behavior were collected from 57 individuals of the study group of wild mandrills of both sexes during 17 months (Nsi Akoué et al., 2017) using five minutes focal samplings of 57 individuals (Altmann, 1974). 


\section{Plant collection and identification}

The plants consumed by the studied mandrills were collected, pressed in the field and authenticated by an expert botanist of the National Herbarium of the Institute of Tropical Medicine and Pharmacopoeia (IPHAMETRA, Libreville, Gabon). Voucher specimens were deposited in the herbarium of the University of Sciences and Techniques of Masuku (Franceville-Gabon).

\section{Nutritional proximate analysis \\ Preparation of sample plants}

The selection of plants used in this study based on the combination of several criteria as consummation frequency by mandrills, their availability in area, their uses by local population (Nsi Akoué et al., 2017; Nsi Akoué, 2017). Principally, the plant parts which were abundantly consumed by mandrills and feebly available in area and also used as food plant by local population were used for nutritional value and antioxidant analysis. Them which were rare in area and abundantly consumed by mandrills and also used in traditional medicine as anti-parasitic or antimicrobial agents (Nsi Akoué, 2017) were used for antimicrobial activities against multi-resistant bacteria from mandrills. Plant parts selected were collected during the behavioural data collection (Nsi Akoué et al., 2017). The samples collected were air-dried at room temperature for a total period six weeks and pulverized into powder using a clean electric blender (Model Phillips 190). The powders of samples were conserved in the flasks until utilization.

\section{Proximate analysis}

The moisture and ash content of samples of plants parts were determined using standard chemical methods as described by Association of Official Analytical Chemists (AOAC, 1990). For moisture, $2 \mathrm{~g}$ of sample was dried at $105^{\circ} \mathrm{C}$ for 24 hours. The ash content was determined by incineration of $2 \mathrm{~g}$ of the sample in a muffle furnace at $550^{\circ} \mathrm{C}$ for 6 hours. Soxhlet extraction technique using petroleum ether $\left(40-50^{\circ} \mathrm{C}\right)$ was used to evaluate the fat content of the sample (Pearson et al., 1981).

Bradford method was used to determine the crude protein content of the sample (Bradford, 1976). The carbohydrate content of the sample was determined by Dubois method (Dubois et al., 1956).

The crude fiber content of the sample has been estimated from the formula (\% carbohydrate $=100 \%$ - sum of percentage of moisture, ash, fat, crude fiber and crude protein contents) (Alinnor and Oze, 2011).

$$
\text { Crude fiber }(\%)=100-[\text { Moisture }(\%)+\text { Fat }(\%)+\text { Carbohydrate }(\%)+\text { Proteine }(\%)]
$$

\section{Determination of total energy}

The total energy of food mandrill was calculated by according the method of Satter et al. (2014) using the formula following:

$$
\text { Energy }(\mathrm{kcal} / 100 \mathrm{~g})=(\% \text { Fat } \times 9.3)+(\% \text { Carbohydrate } \times 4.1)+(\% \text { Proteine } \times 4.1)
$$

\section{Mineral Analysis}

The mineral contents of the samples were estimated by the wet digestion method (AOAC, 1995). Briefly, $1 \mathrm{~g}$ of the powdered plant sample was mixed with a10 $\mathrm{mL}$ of nitric acid, $\mathrm{HNO} 3(100 \%)$ in a Becher. The digestion was carried out at low heat until the brown fumes had been given off, and heating continued until all the solids dissolved and the appearance of white fumes. Then we added $3 \mathrm{~mL}$ of perchloric acid, $\mathrm{HClO} 4(70 \%)$ and continued the digestion until the reduction of bulk. After cooling, the digest was filtered and transferred into $100 \mathrm{~mL}$ volumetric flask and made up to the mark with distilled water. The solution was used for the estimation of minerals. The $\mathrm{K}, \mathrm{Ca}$ and $\mathrm{Na}$ were estimated by flame photometer (PFP76 Flame Photometer, Jenway), while $\mathrm{Zn}, \mathrm{Fe}, \mathrm{Mg}$ and $\mathrm{Cu}$ were determined using atomic absorption spectrophotometer (Model AA240FS, Varian). Phosphorus was determined by the Vanado Molybdate-Yellow method (AOAC, 2000).

\section{Phytochemical analysis}

\section{Preparation of plant extract}

The plant parts consumed by the mandrillswere collected during the Behavioural data collection (Nsi Akoué et al., 2017). Them samples collected were air-dried at room temperature for a total period six weeks and pulverized to powder using a clean electric blender (Model Phillips 190). A $25 \mathrm{~g}$ sample of the pulverized of each plant parts was lyophilized and the extract obtained is stored in vials protected from light until the completion of various tests. 


\section{Phytochemical screening}

Phytochemical tests the dry extracts (ethanolic, ethanolic-water and aqueous) bioactive compounds was realized using standard procedures (Bruneton, 1999; Bidié et al.,2008), with small modifications (Nsi Akoué et al., 2013; Nsi Akoué, 2017). Qualitative analysis tests were realized for the presence of chemical constituents such as sterols, terpenoids, tannins, polyphenols, flavonoids, quinones and saponins. The alkaloids were tested by the method of Dragendorff and Meyer (Bruneton, 1999).

\section{Total Phenols content extracts}

The Folin-Ciocalteu method was used to measure total amount of total phenols content (Singleton et al., 1999). Aliquots of $0.25 \mathrm{~mL}$ of each leaf extracts $(1 \mathrm{mg} / \mathrm{mL})$ were mixed with $1.25 \mathrm{~mL}$ Folin-Ciocalteu reagent $(0.2 \mathrm{~N}$ diluted in methanol). A reagent blank using methanol instead of sample was prepared. After 5 min incubation at room temperature, $1 \mathrm{~mL}$ sodium carbonate solution $(7.5 \%)$ was added. Samples were incubated at room temperature for $1 \mathrm{~h}$ and the absorbances were measured at $765 \mathrm{~nm}$ versus the prepared blank.

All tests were carried out in triplicate and total phenols content was expressed as $\mathrm{mg}$ of Gallic acid equivalents (GAE) per $100 \mathrm{~g}$ of drug.

\section{Total flavonoids content extracts}

The Dowd method was used to measure total amount of flavonoids (Arvouet-Grand et al., 1994) with slightly modification. Quercetin was used as standard. $1 \mathrm{~mL}$ of $2 \% \mathrm{AlCl} 3$ in methanol was added to $1 \mathrm{~mL}$ of plant extract (1 $\mathrm{mg} / \mathrm{mL})$. After $10 \mathrm{~min}$ of incubation at room temperature $\left(20^{\circ} \mathrm{C}\right)$, absorbances were read at $415 \mathrm{~nm}$. All tests were carried out in triplicate and results were expressed as Quercetin equivalent (QE).

\section{Total tannins content extracts}

The reference method of European Community was used to measure total amount of tannins (1994) with small modifications. Aliquots of $250 \mu \mathrm{l}$ of extract $(1 \mathrm{mg} / \mathrm{mL})$ were added at 250 of $\mu \mathrm{l}$ ammonium citrate ferric reagent $(3.5 \mathrm{~g} / \mathrm{L})$ more $250 \mu \mathrm{l}$ of ammonia $(20 \%, 8 \mathrm{~g} / \mathrm{L})$ and $1200 \mu \mathrm{l}$ of $\mathrm{H}_{2} \mathrm{O}$. After $10 \mathrm{~min}$ of incubation at room temperature $\left(20^{\circ} \mathrm{C}\right)$, the absorbances were read at $525 \mathrm{~nm}$. All tests were carried out in triplicate and results were expressed as Tannic acid equivalent (EAT).

\section{Antioxidant activity index determination}

The Antioxidant Activity Index (AAI) was determinate according to the radical 2,2-diphenyl-1-picrylhydrazyl (DPPH) method as described by Scherer and Godoy, (2009). Briefly, the working reagent was prepared by dissolving $10 \mathrm{mg}$ of DPPH in $100 \mathrm{~mL}$ ethanol. Graded concentrations of extracts ranging from 0.781 to $100 \mu \mathrm{g} / \mathrm{mL}$ obtained by two-fold dilutions were prepared and $100 \mu \mathrm{L}$ of each dilution were mixed with $100 \mu \mathrm{L}$ of the extract solution. Absorbences were measured at $517 \mathrm{~nm}$ after $15 \mathrm{~min}$ incubation at room temperature in the dark. Ascorbic acid (Vitamin C) and Quercetin were used as references. The ability to scavenge DPPH radical was calculated by the following equation:

$$
\mathrm{RSA}=[(\text { Abs (control) }-(\text { Abs (sample) })] / \text { Abs (control) }) \times 100
$$

With, RSA is the percentage of free radical scavenging activity. The $\mathrm{IC}_{50}$ (concentration providing $50 \%$ inhibition) of extracts and standards was determinate using regression curves in the linear range of concentrations. The antioxidant activity index was calculated as following formula

Where [DPPH $(\mu \mathrm{g} / \mathrm{mL})]$, is the final concentration of DPPH.

$$
\text { AAI }=[\mathrm{DPPH}(\mu \mathrm{g} / \mathrm{mL})] / \mathrm{IC}_{50}(\mu \mathrm{g} / \mathrm{mL})
$$

$[D P P H]$ is the final concentration of DPPH. We considered criteria of Scherer and Godoy according to which plant extracts show poor antioxidant activity when AAI $<0.5$, moderate antioxidant activity when AAI between 0.5 and 1.0, strong antioxidant activity when AAI between 1.0 and 2.0, and very strong when AAI $>2.0$.

\section{Antimicrobial studies}

\section{Test microorganisms}

Microorganisms strains used in this study were principally multiresistant bacteria strains of mandrills from Bacteriology Laboratory of Centre International de Recherche Médicale de Franceville (CIRMF).These isolates were constituted byCitrobacter youngae (CY95, CY127), Escherichia coli (EC114, EC121, EC129 and EC292), Enterobacter cloacae complex (ECC110), Klebsiella pneumoniae ssp pneumonia (KPP32) and Pseudomonas aeruginosa (PA102 and PA383). These isolates were resistant at three standard antibiotics such as Cefotaxime, 
Ceftazidime and Ertapenem. The selection of this these antibiotics depended on their availability and resistance to some antibiotics used in the treatment of human microbial infections. Thus microorganisms, well represented, are opportunists pathogens most frequently implicated in infectious diseases in tropical areas (Arvouet-Grant et al., 1994; Koudou et al., 2008).

\section{Determination inhibition zone diameters}

The assay was conducted as described by Bssaibis et al., (2009). Briefly, some bacteria strains from growth on Macconkey agar medium incubated at $37{ }^{\circ} \mathrm{C}$ for $24 \mathrm{~h}$ were suspended in saline solution $0.9 \% \mathrm{NaCl}$ and adjusted to a turbidity of $0.5 \mathrm{McFarland}$ standards(108 CFU/ml) (Lennette et al., 1981). Each suspension was used to inoculate into a Petri dish $90 \mathrm{~mm}$ diameter using a sterile swab (DeltaLab). Then, $6 \mathrm{~mm}$ wells were dug into the agar of each Petri dish with a sterile cork borer. And $50 \mu \mathrm{L}$ of each plant extract tested at $2000 \mu \mathrm{g} / \mathrm{mL}$ were put in these wells. The dissolution of the extract was added by $0.5 \%$ (v/v) DMSO which did not affect microorganism growth, according to our control experiments. Diffusion discs of Cefotaxime (CTX $30 \mu \mathrm{g}$ ), Ceftazidime (CAZ $10 \mu \mathrm{g}$ ), and Ertapenem (ERT $10 \mu \mathrm{g}$ ), (Bio-Rad) were used as positive reference standard to determine the sensitivity of the strains. Plates were incubated aerobically at $37^{\circ} \mathrm{C}$ for $24 \mathrm{~h}$. The antimicrobial activities were evaluated by measuring the diameters of the inhibition zones with a graduated rule.

\section{Determination the Minimum Inhibitory Concentration by Broth Dilution Assay}

The microdilution method was used to determine minimal inhibitory concentrations (MIC) and minimal bactericidal concentrations (MBC) of the extract against the test microorganisms as described by Bssaibis et al., (2009). The extracts dissolved in $0.5 \%$ DMSO were transferred in tubes to obtain a two-fold serial dilutions ranging from 4.8 to $2500 \mu \mathrm{g} / \mathrm{mL}$. The tubes were inoculated in air with microbial suspensions at $37^{\circ} \mathrm{C}$ for 24 hours. MIC was recorded as a lowest extract concentration demonstrating no visible growth in the broth. MBC was recorded as a lowest extract concentration that kills $99.9 \%$ of bacterial inoculate. MBC values were determined by removing $100 \mu$ of bacterial suspension for subculture demonstrating no visible growth and by inoculating nutrient agar plates. Plates were incubated at $37^{\circ} \mathrm{C}$ for a total period of $48 \mathrm{~h}$ (Obame et al., 2007, Koudou et al., 2008).

\section{Results:-}

\section{Nutritional composition of wild vegetables}

Results of analysis of nutrient content (Table 1) of 22 wild vegetables from mandrill diet and also used by native population show that the crude protein contents of the examined vegetables were ranged from $1.7 \pm 0.0 \%$ to $34.6 \pm$ $0.7 \%$ of dry matter (DM) basis and the mean of protein content was $12,55 \pm 1,42 \% \mathrm{DM}$. The high value of protein was obtained with Ricinodendron heudelotii (34.6 $\pm 0.7 \%$ DM), Pentaclethra macrophylla $(20.9 \pm 3.6 \%$ DM), followed by Landolphia mannii (19.9 \% DM), Uapaca guineensis $(19.3 \pm 0.1 \% \mathrm{DM})$ and Aframomum alboviolaceum $(17.8 \pm 3.3 \%$ DM). The small quantity was obtained with Cnestis $s p(1.7 \pm 0.0 \%$ DM), followed of Asplenium africanum $(2.8 \pm 0.3 \% \mathrm{DM})$ and Renealmia macrocolea $(5.8 \pm 5.1 \% \mathrm{DM})$.

Concerning the fats, values were ranged from $0.8 \pm 0.9 \%$ to $33.7 \pm 0.7 \% \mathrm{DM}$ and the mean value was $12.40 \pm$ $3.11 \%$ DM. The high values were obtained with Landolphia mannii $(49.2 \pm 0.3 \%$ DM) followed of Pentaclethra macrophylla (41.6 $\pm 0.4 \% \mathrm{DM})$ and Aframomum daniellii $(23.1 \pm 3.4 \% \mathrm{DM})$. Tristemma mauritianum on the other hand has presented the small value of fat $(0.8 \pm 0.9 \%$ DM resp. $)$ and average of fat content was $8.83 \pm 2.05 \%$ DM.

Vegetables average of carbohydrate content was $16.48 \pm 3.63 \% \mathrm{DM}$ and the carbohydrate content were ranged from $0.6 \pm 1.7$ to $78.3 \pm 2.1 \%$ DM. The higher values were obtained with Chrysophyllum africanum $(78.3 \pm 2.1 \% \mathrm{DM})$, Chrysophyllum lacourtianum $(58.1 \pm 3.0 \% \mathrm{DM})$ and Landolphia hirsuta $(69.7 \pm 0.8 \% \mathrm{DM})$. The small carbohydrate content was obtained with Macaranga schweinfurthii $(3.7 \pm 1.6 \% \mathrm{DM})$.

The mean of moisture content was $35.18 \pm 4.99 \% \mathrm{DM}$ and the values were found between $3.6 \pm 0.8$ and $88.5 \pm 0.7 \%$ DM. Mean of ash content was $5.92 \pm 0.68 \% \mathrm{DM}$ and the values were ranged between $2.7 \pm 0.3$ and $11.7 \pm 0.9 \%$ DM.

The results obtained on caloric values of vegetables ranged between $53.07 \pm 3.9$ and $608.27 \pm 3.0 \mathrm{kcal} / 100 \mathrm{~g}$ DM with an average of $229.89 \pm 30.29 \mathrm{kcal} / 100 \mathrm{~g}$ DM. The high caloric values were obtained with Ricinodendron heudelotii $(608.27 \pm 3.0 \mathrm{kcal} / 100 \mathrm{~g} \mathrm{DM})$ followed of Pentaclethra macrophylla $(533.25 \pm 0.9 \mathrm{kcal} / 100 \mathrm{~g} \mathrm{DM})$ and Chrysophyllum africanum $(387.18 \pm 4.3 \mathrm{kcal} / 100 \mathrm{~g} \mathrm{DM})$. The small caloric values were obtained with the petioles of Macaranga schweinfurthii $(75.620 \pm 2.4 \mathrm{kcal} / 100 \mathrm{~g} \mathrm{DM})$. 
The crude fiber content of vegetables was found between $1.4 \pm 2.1 \%$ and $41 \pm 1.0 \%$ DM and their mean was of $10.55 \pm 1.8 \% \mathrm{DM}$.

Table 1:-Nutritional composition of the some wild plants from Mandrillus sphinx diet (\%).

\begin{tabular}{|c|c|c|c|c|c|c|c|}
\hline Vegetal species & $\begin{array}{c}\text { Pla } \\
\text { nt } \\
\text { part } \\
\text { s }\end{array}$ & $\begin{array}{l}\text { Moistu } \\
\text { re }\end{array}$ & Ash & $\begin{array}{c}\text { Carbo- } \\
\text { hydrates }\end{array}$ & Fat & Protein & $\begin{array}{c}\text { Energy (Kcal/100g } \\
\text { DM) }\end{array}$ \\
\hline $\begin{array}{l}\text { Aframoтит } \\
\text { alboviolaceum }\end{array}$ & $\begin{array}{l}\text { Frui } \\
\mathrm{t}\end{array}$ & $\begin{array}{c}9.9 \pm \\
2.4\end{array}$ & $\begin{array}{c}2.66 \pm \\
0.3\end{array}$ & $8.89 \pm 0.2$ & $\begin{array}{c}7.21 \pm \\
3.1\end{array}$ & $\begin{array}{c}17.8 \pm \\
1.1\end{array}$ & $176.65 \pm 2.6$ \\
\hline $\begin{array}{l}\text { Aframomum } \\
\text { polyanthum }\end{array} \quad c f$ & $\begin{array}{l}\text { Frui } \\
\mathrm{t}\end{array}$ & $\begin{array}{c}7.5 \pm \\
3.2\end{array}$ & $\begin{array}{c}3.67 \pm \\
3.2\end{array}$ & $6.01 \pm 3.9$ & $\begin{array}{c}6.60 \pm \\
0,1\end{array}$ & $\begin{array}{c}5.78 \pm \\
4.8\end{array}$ & $109.72 \pm 6.8$ \\
\hline Aframomum daniellii & $\begin{array}{l}\text { Frui } \\
\mathrm{t}\end{array}$ & $\begin{array}{c}8.80 \pm \\
4.7\end{array}$ & $\begin{array}{l}9.70 \pm \\
5.1\end{array}$ & $11.10 \pm 1.6$ & $\begin{array}{c}23.1 \pm \\
3.4\end{array}$ & $\begin{array}{c}8.30 \pm \\
6.1\end{array}$ & $294.37 \pm 3.1$ \\
\hline Aframomum $s p$ & $\begin{array}{l}\text { Frui } \\
t\end{array}$ & $\begin{array}{l}26.0 \pm \\
2.3\end{array}$ & $\begin{array}{c}5.04 \pm \\
1.3\end{array}$ & $9.59 \pm 4.3$ & $\begin{array}{l}4,30 \pm \\
2.3\end{array}$ & $\begin{array}{c}16.6 \pm \\
3.3\end{array}$ & $147.37 \pm 1.7$ \\
\hline Alchornea floribunda & $\begin{array}{l}\text { Frui } \\
\mathrm{t}\end{array}$ & $\begin{array}{c}74.5 \pm \\
0.0\end{array}$ & $\begin{array}{c}4.06 \pm \\
0.0\end{array}$ & $7.63 \pm 1.9$ & $\begin{array}{c}18.9 \pm \\
3.6\end{array}$ & $\begin{array}{c}15.8 \pm \\
2.9\end{array}$ & $271.83 \pm 4.1$ \\
\hline $\begin{array}{l}\text { Chrysophyllum } \\
\text { africanum }\end{array}$ & $\begin{array}{l}\text { Frui } \\
t\end{array}$ & $\begin{array}{c}6.7 \pm \\
0.2\end{array}$ & $\begin{array}{l}2.7 \pm \\
0.7\end{array}$ & $78.3 \pm 2.1$ & $\begin{array}{c}4.6 \pm \\
3.2\end{array}$ & $\begin{array}{l}5.7 \pm \\
0.01\end{array}$ & $387.18 \pm 4.3$ \\
\hline $\begin{array}{l}\text { Chrysophyllum } \\
\text { lacourtianum }\end{array}$ & $\begin{array}{l}\text { Frui } \\
\mathrm{t}\end{array}$ & $\begin{array}{c}9.4 \pm \\
0.9\end{array}$ & $6 \pm 2.1$ & $58.1 \pm 3.0$ & $3 \pm 0.91$ & $\begin{array}{c}12.3 \pm \\
2.1 \\
\end{array}$ & $316.54 \pm 3.9$ \\
\hline Landolphia mannii & $\begin{array}{l}\text { Frui } \\
\mathrm{t}\end{array}$ & $\begin{array}{c}11.2 \pm \\
0.5\end{array}$ & $\begin{array}{c}8.1 \pm \\
1.7\end{array}$ & $5.0 \pm 2.5$ & $\begin{array}{c}49.2 \pm \\
0.3\end{array}$ & $\begin{array}{c}19.9 \pm \\
1.9\end{array}$ & $327.40 \pm 4.3$ \\
\hline Lasianthus batangensis & $\begin{array}{l}\text { Frui } \\
t\end{array}$ & $\begin{array}{c}63.3 \pm \\
0.0\end{array}$ & $\begin{array}{l}7.7 \pm \\
0.31\end{array}$ & $5.2 \pm 0.12$ & $\begin{array}{c}4.7 \pm \\
7.1\end{array}$ & $9.21 \pm 0.0$ & $102.96 \pm 1.9$ \\
\hline $\begin{array}{l}\text { Macaranga } \\
\text { schweinfurthii }\end{array}$ & $\begin{array}{l}\text { Roo } \\
t\end{array}$ & $28 \pm 1.4$ & $\begin{array}{c}3.7 \pm \\
0.3\end{array}$ & $3.7 \pm 1.6$ & $\begin{array}{l}3.6 \pm \\
0.09\end{array}$ & $1.7 \pm 0.0$ & $75.620 \pm 2.4$ \\
\hline Maesobotrya staudtii & $\begin{array}{l}\text { Frui } \\
\mathrm{t}\end{array}$ & $\begin{array}{c}71.4 \pm \\
0.0\end{array}$ & $\begin{array}{c}4.3 \pm \\
1.0\end{array}$ & $6.9 \pm 0.37$ & $\begin{array}{c}2.6 \pm \\
0.0\end{array}$ & $17.8 \pm 0.0$ & $125.61 \pm 0.9$ \\
\hline Medinilla mirabilis & $\begin{array}{l}\text { Frui } \\
t\end{array}$ & $\begin{array}{c}28.4 \pm \\
2.1\end{array}$ & $4 \pm 0.0$ & $4.34 \pm 1.6$ & $\begin{array}{c}2.8 \pm \\
3.2\end{array}$ & $\begin{array}{c}22.1 \pm \\
0.0\end{array}$ & $134.44 \pm 1.1$ \\
\hline $\begin{array}{l}\text { Megaphrynium } \\
\text { macrostachum }\end{array}$ & $\begin{array}{l}\text { See } \\
d\end{array}$ & $\begin{array}{c}7.5 \pm \\
1.0\end{array}$ & $9.9 \pm 0.1$ & $1.18 \pm 2.8$ & $\begin{array}{c}33.7 \pm \\
0.7\end{array}$ & $\begin{array}{l}6.3 \pm \\
0.09\end{array}$ & $344.08 \pm 4.0$ \\
\hline $\begin{array}{l}\text { Microdesmis } \\
\text { haumaniana }\end{array}$ & $\begin{array}{l}\text { Frui } \\
\mathrm{t}\end{array}$ & $\begin{array}{c}71.4 \pm 0 . \\
0\end{array}$ & $3 \pm 0.03$ & $11.8 \pm 0.2$ & $3.9 \pm 0.0$ & $\begin{array}{c}11.9 \pm 0.5 \\
1\end{array}$ & $133.44 \pm 1.5$ \\
\hline Palisota ambigua & $\begin{array}{l}\text { Frui } \\
\mathrm{t}\end{array}$ & $\begin{array}{l}72 \pm \\
0.34 \\
\end{array}$ & $\begin{array}{c}11.7 \pm \\
0.9\end{array}$ & $12.7 \pm 1.0$ & $\begin{array}{l}2.1 \pm \\
0.01\end{array}$ & $\begin{array}{c}22.4 \pm \\
0.0\end{array}$ & $163.44 \pm 0.7$ \\
\hline Paspalum scrobiculatum & $\begin{array}{l}\text { Ste } \\
\mathrm{m}\end{array}$ & $\begin{array}{c}88.5 \pm \\
0.7 \\
\end{array}$ & $\begin{array}{c}5.7 \pm \\
0.3 \\
\end{array}$ & $17.7 \pm 5.0$ & $\begin{array}{c}3.4 \pm \\
0.5 \\
\end{array}$ & $\begin{array}{c}10.1 \pm \\
0.0\end{array}$ & $145.60 \pm 4.9$ \\
\hline $\begin{array}{l}\text { Pentaclethra } \\
\text { macrophylla }\end{array}$ & $\begin{array}{l}\text { See } \\
\mathrm{d}\end{array}$ & $\begin{array}{c}11.9 \pm \\
1.0\end{array}$ & $3 \pm 0.91$ & $14.8 \pm 2.1$ & $\begin{array}{c}41.6 \pm \\
0.4\end{array}$ & $\begin{array}{c}20.9 \pm \\
3.6\end{array}$ & $533.25 \pm 0.9$ \\
\hline $\begin{array}{l}\text { Pseudospondias } \\
\text { longifolia }\end{array}$ & $\begin{array}{l}\text { Frui } \\
\mathrm{t}\end{array}$ & $\begin{array}{c}46.1 \pm \\
0.3\end{array}$ & $3.10 \pm 2$ & $9.65 \pm 0.3$ & $\begin{array}{c}9.8 \pm \\
0.9 \\
\end{array}$ & $\begin{array}{c}11.48 \pm \\
1.1 \\
\end{array}$ & $177.77 \pm 3.9$ \\
\hline $\begin{array}{l}\text { Rhynchospora } \\
\text { corymbosa }\end{array}$ & $\begin{array}{l}\text { Frui } \\
t\end{array}$ & $\begin{array}{c}57.3 \pm \\
0.6\end{array}$ & $\begin{array}{l}5.3 \pm \\
2.4\end{array}$ & $9.9 \pm 0.9$ & $\begin{array}{c}2.6 \pm \\
2.6\end{array}$ & $\begin{array}{c}16.18 \pm \\
0.2\end{array}$ & $133.65 \pm 2.9$ \\
\hline $\begin{array}{l}\text { Ricinodendron } \\
\text { heudelotii }\end{array}$ & $\begin{array}{l}\text { See } \\
\mathrm{d}\end{array}$ & $\begin{array}{c}5.6 \pm \\
0.0\end{array}$ & $\begin{array}{c}11.3 \pm \\
0.1\end{array}$ & $32.1 \pm 0.4$ & $36 \pm 0.6$ & $\begin{array}{c}34.6 \pm \\
0.7\end{array}$ & $608.27 \pm 3.0$ \\
\hline Tristemma mauritianum & Leaf & $\begin{array}{c}34.6 \pm \\
3.1 \\
\end{array}$ & $\begin{array}{c}3.6 \pm \\
0.2\end{array}$ & $9.67 \pm 1.9$ & $\begin{array}{l}1.7 \pm \\
0.39 \\
\end{array}$ & $\begin{array}{c}16.9 \pm \\
0.0\end{array}$ & $124.93 \pm 5.2$ \\
\hline Uapaca guineensis & $\begin{array}{l}\text { Frui } \\
\mathrm{t}\end{array}$ & $\begin{array}{c}74.7 \pm 0 . \\
0\end{array}$ & $4 \pm 2.9$ & $18.4 \pm 1.2$ & $\begin{array}{c}7.4 \pm \\
0.0\end{array}$ & $\begin{array}{c}19.3 \pm \\
0.1 \\
\end{array}$ & $223.39 \pm 3.1$ \\
\hline
\end{tabular}

All data were expressed as mean \pm SD of triplicate experiment $(n=3)$. 
Minerals composition of the wild vegetables consumed by mandrills.

The mineral compositions of 22 wild vegetables from mandrill diet such as potassium (K), calcium (Ca), sodium $(\mathrm{Na})$, magnesium $(\mathrm{Mg})$, phosphorus $(\mathrm{P})$, iron $(\mathrm{Fe})$, zinc $(\mathrm{Zn})$, and copper $(\mathrm{Cu})$ are presented in Table 2.

The results show that the high mineral content was obtained with the majority of the organ of plants eaten. The means of the mineral content of vegetables were $1663.2 \mathrm{mg} / \mathrm{kg}$ of dry matter $(\mathrm{Ca}), 1199.82 \mathrm{mg} / \mathrm{kg}$ dry matter $(\mathrm{Mg})$, $127733.05 \mathrm{mg} / \mathrm{kg}$ dry matter $(\mathrm{K}), 2698.64 \mathrm{mg} / \mathrm{kg}$ dry matter $(\mathrm{P}), 8459.2 \mathrm{mg} / \mathrm{kg}$ dry matter $(\mathrm{Na}), 369.39 \mathrm{mg} / \mathrm{kg}$ dry matter $(\mathrm{Zn}), 171.86 \mathrm{mg} / \mathrm{kg}$ dry matter $(\mathrm{Cu})$ and $1187.26 \mathrm{mg} / \mathrm{kg}$ dry matter $(\mathrm{Fe})$.

The mineral content of 22 vegetables varies of $11.92 \pm 4.1$ and $10483.2 \pm 1.4 \mathrm{mg} / \mathrm{kg}$ dry matter $(\mathrm{Fe})$, of $29.1 \pm 0.01$ and $362.91 \pm 2.1 \mathrm{mg} / \mathrm{kg}$ dry matter $(\mathrm{Cu})$, of $105.98 \pm 0.8$ and $912.64 \pm 2.3 \mathrm{mg} / \mathrm{kg}$ dry matter $(\mathrm{Zn})$, of $1139.4 \pm 2.1$ and $15885.1 \pm 1.0 \mathrm{mg} / \mathrm{kg}$ dry matter $(\mathrm{Na}), 970 \pm 7.1$ and $7110 \pm 3.1 \mathrm{mg} / \mathrm{kg}$ dry matter $(\mathrm{P})$, of $355.54 \pm 6.0$ and $220424.6 \pm 3.1 \mathrm{mg} / \mathrm{kg}$ dry matter $(\mathrm{K}), 198 \pm 2.0$ and $3470 \pm 3.4$ and $\mathrm{mg} / \mathrm{kg}$ dry matter $(\mathrm{Mg})$ and of $610 \pm 1.5$ and $2310 \pm 2.4 \mathrm{mg} / \mathrm{kg}$ dry matter $(\mathrm{Ca})$.

Our results shown also that ratio $\mathrm{Na} / \mathrm{K}$ of 22 plant species analyzed was ranged from 0.01 (Aframomum alboviolaceum and Macaranga schweinfurthii) to 2.36 (Palisota ambigua) and the ration $\mathrm{Zn} / \mathrm{Cu}$ was from 0.75 (Aframomum cf polyanthum) to 6.79 (Aframomum daniellii) (Table 2).

Table2:-Crud fiber (\%) and mineral concentration in the wild vegetables (mg/kg DM).

\begin{tabular}{|c|c|c|c|c|c|c|c|c|c|c|}
\hline $\begin{array}{l}\text { Vegetal } \\
\text { species }\end{array}$ & $\begin{array}{c}\text { Crud } \\
\text { Fibe } \\
r\end{array}$ & $\mathrm{Ca}$ & $\mathrm{Mg}$ & K & $\mathbf{P}$ & $\mathrm{Na}$ & $\mathrm{Zn}$ & $\mathrm{Cu}$ & Fe & $\begin{array}{c}\mathrm{Na} / \\
\mathrm{K}\end{array}$ \\
\hline $\begin{array}{l}\text { A. } \\
\text { alboviolaceu } \\
m\end{array}$ & $\begin{array}{c}2.1 \pm \\
1.5\end{array}$ & $\begin{array}{l}1650 \\
\pm 0.8\end{array}$ & $\begin{array}{l}219 \\
0 \pm \\
2.0 \\
\end{array}$ & $\begin{array}{c}204462 \\
5 \pm 0.8\end{array}$ & $\begin{array}{l}710 \\
0 \pm \\
1,6\end{array}$ & $\begin{array}{c}1272.4 \\
\pm 2.4\end{array}$ & $\begin{array}{c}324.50 \\
\pm 2.4\end{array}$ & $\begin{array}{c}52.80 \pm \\
1.6\end{array}$ & $\begin{array}{c}269.23 \pm \\
0.9\end{array}$ & 0.01 \\
\hline $\begin{array}{l}A . \\
\text { polyanthum }\end{array}$ & $\begin{array}{c}2.9 \pm \\
0.4\end{array}$ & $\begin{array}{c}1200 . \\
4 \pm \\
1.1\end{array}$ & $\begin{array}{r}210 \\
\pm 8.1\end{array}$ & $\begin{array}{c}92400.0 \\
\pm 2.3\end{array}$ & $\begin{array}{l}139 \\
0 \pm \\
1.0\end{array}$ & $\begin{array}{c}5467.2 \\
\pm 2.3\end{array}$ & $\begin{array}{c}136.62 \\
\pm 0.2\end{array}$ & $\begin{array}{c}182.16 \\
\pm 4.1\end{array}$ & $\begin{array}{c}4015.38 \\
\pm 1.5\end{array}$ & 0.06 \\
\hline A. daniellii & $\begin{array}{c}13.1 \\
\pm 3\end{array}$ & $\begin{array}{c}610 \pm \\
1.5\end{array}$ & $\begin{array}{l}125 \\
8 \pm \\
4.7 \\
\end{array}$ & $\begin{array}{c}4316 \pm \\
2.1\end{array}$ & $\begin{array}{l}618 \\
0 \pm \\
1.0\end{array}$ & $\begin{array}{c}1139.4 \\
\pm 2.1\end{array}$ & $\begin{array}{c}177.2 \pm \\
1.2\end{array}$ & $\begin{array}{c}29.1 \pm \\
0.01\end{array}$ & $\begin{array}{c}276 \pm \\
1.2\end{array}$ & 0.26 \\
\hline A. floribunda & $\begin{array}{c}3.3 \pm \\
1.9\end{array}$ & $\begin{array}{l}1860 \\
\pm 2.3\end{array}$ & $\begin{array}{c}280 \\
\pm \\
2.7 \\
\end{array}$ & $\begin{array}{c}195525 \\
\pm 1.8\end{array}$ & $\begin{array}{l}330 \\
0 \pm \\
9.0\end{array}$ & $\begin{array}{c}21666 \\
\pm 1.7\end{array}$ & $\begin{array}{c}817.65 \\
\pm 0.1\end{array}$ & $\begin{array}{c}337.7 \pm \\
1.6\end{array}$ & $\begin{array}{c}41.43 \pm \\
3.0\end{array}$ & 0.11 \\
\hline $\begin{array}{l}\text { Aframomum } \\
\text { sp }\end{array}$ & $\begin{array}{c}10.2 \\
\pm 6\end{array}$ & $\begin{array}{l}1940 \\
\pm 1.8\end{array}$ & $\begin{array}{l}201 \\
0 \pm \\
1.1\end{array}$ & $\begin{array}{c}209163 \\
3 \pm 0.4\end{array}$ & $\begin{array}{l}250 \\
0 \pm \\
0,1\end{array}$ & $\begin{array}{c}4301.5 \\
\pm 1.1\end{array}$ & $\begin{array}{c}565.92 \\
\pm 3.1\end{array}$ & $\begin{array}{c}146.72 \\
\pm 1.3\end{array}$ & $\begin{array}{c}36.64 \pm \\
2.6\end{array}$ & 0.02 \\
\hline C. africanum & $\begin{array}{c}21 \pm \\
1.2\end{array}$ & $\begin{array}{l}1690 \\
\pm 0.6\end{array}$ & $\begin{array}{c}101 \\
0 \\
\pm 2.0 \\
\end{array}$ & $\begin{array}{c}12720.4 \\
\pm 2.8\end{array}$ & $\begin{array}{l}236 \\
0 \pm \\
1.3 \\
\end{array}$ & $\begin{array}{c}3822.0 \\
\pm 1.0\end{array}$ & $\begin{array}{c}114.30 \\
\pm 3.4\end{array}$ & $\begin{array}{c}98.34 \pm \\
3.1\end{array}$ & $\begin{array}{c}97.62 \pm \\
3.4\end{array}$ & 0.30 \\
\hline $\begin{array}{l}\text { C. } \\
\text { lacourtianum }\end{array}$ & $\begin{array}{c}13.2 \\
\pm 2\end{array}$ & $\begin{array}{l}1970 \\
\pm 3.1\end{array}$ & $\begin{array}{c}198 \\
\pm \\
2.0\end{array}$ & $\begin{array}{c}30124.3 \\
\pm 3.8\end{array}$ & $\begin{array}{c}970 \\
\pm \\
7.1 \\
\end{array}$ & $\begin{array}{c}2927.9 \\
\pm 6.0\end{array}$ & $\begin{array}{c}234.50 \\
\pm 3.4\end{array}$ & $\begin{array}{c}118.64 \\
\pm 2.7\end{array}$ & $\begin{array}{c}112.1 \pm \\
6.1\end{array}$ & 0.10 \\
\hline $\begin{array}{l}\text { L. } \\
\text { batangensis }\end{array}$ & $\begin{array}{c}18.1 \\
\pm 1\end{array}$ & $\begin{array}{l}1750 \\
\pm 2.2\end{array}$ & $\begin{array}{c}117 \\
0 \\
\pm 4.3 \\
\end{array}$ & $\begin{array}{c}82705.0 \\
\pm 1.6\end{array}$ & $\begin{array}{l}361 \\
0 \pm \\
2.0 \\
\end{array}$ & $\begin{array}{l}7624.3 \\
\pm 1.10\end{array}$ & $\begin{array}{c}439.94 \\
\pm 0.2\end{array}$ & $\begin{array}{c}152.21 \\
\pm 1.8\end{array}$ & $\begin{array}{c}145.80 \pm \\
0.1\end{array}$ & 0.09 \\
\hline L. mannii & $\begin{array}{c}11 \pm \\
1.2\end{array}$ & $\begin{array}{l}2310 \\
\pm 0.6\end{array}$ & $\begin{array}{l}283 \\
0 \pm \\
3.0\end{array}$ & $\begin{array}{c}178435 \\
8 \pm 2.0\end{array}$ & $\begin{array}{l}126 \\
0 \pm \\
3.1\end{array}$ & $\begin{array}{c}1418.3 \\
\pm 1.9\end{array}$ & $\begin{array}{c}654.32 \\
\pm 1.3\end{array}$ & $\begin{array}{c}362.91 \\
\pm 2.1\end{array}$ & $\begin{array}{c}1835.31 \\
\pm 1.1\end{array}$ & 0.01 \\
\hline
\end{tabular}




\begin{tabular}{|c|c|c|c|c|c|c|c|c|c|c|}
\hline $\begin{array}{l}\text { M. } \\
\text { haumaniana }\end{array}$ & $\begin{array}{c}7.0 \pm \\
1.5\end{array}$ & $\begin{array}{l}1120 \\
\pm 1.3\end{array}$ & $\begin{array}{l}261 \\
0 \pm \\
1.4\end{array}$ & $\begin{array}{c}204143 \\
8 \pm 2.9\end{array}$ & $\begin{array}{l}125 \\
0 \pm \\
2.1\end{array}$ & $\begin{array}{l}15885 \\
1 \pm 1.0\end{array}$ & $\begin{array}{c}185.34 \\
\pm 0.1\end{array}$ & $\begin{array}{c}190.8 \pm \\
2.5\end{array}$ & $\begin{array}{c}128.32 \pm \\
2.1\end{array}$ & 0.08 \\
\hline $\begin{array}{l}\text { M. } \\
\text { macrostachu } \\
m\end{array}$ & $\begin{array}{c}31 \pm \\
3.4\end{array}$ & $\begin{array}{l}2010 \\
\pm 1.2\end{array}$ & $\begin{array}{c}650 \\
\pm \\
2.5\end{array}$ & $\begin{array}{c}130972 . \\
9 \pm 7.0\end{array}$ & $\begin{array}{l}127 \\
0 \pm \\
0.8\end{array}$ & $\begin{array}{c}3565.3 \\
\pm 0.8\end{array}$ & $\begin{array}{l}400.58 \\
\pm 2.6\end{array}$ & $\begin{array}{c}79.63 \pm \\
6.1\end{array}$ & $\begin{array}{c}1370.6 \pm \\
2.3\end{array}$ & 0.03 \\
\hline M. mirabilis & $\begin{array}{c}4.7 \pm \\
0.4\end{array}$ & $\begin{array}{l}1800 \\
\pm 0.6\end{array}$ & $\begin{array}{l}164 \\
0 \pm \\
0.1\end{array}$ & $\begin{array}{c}135225 \\
8 \pm 1.7\end{array}$ & $\begin{array}{l}184 \\
0 \pm \\
0.6\end{array}$ & $\begin{array}{l}3336.6 \\
\pm 1.01\end{array}$ & $\begin{array}{c}234.07 \\
\pm 2.5\end{array}$ & $\begin{array}{c}74.96 \pm \\
2.0\end{array}$ & $\begin{array}{c}10483.2 \\
2 \pm 1.4\end{array}$ & 0.02 \\
\hline $\begin{array}{l}\text { M. } \\
\text { schweinfurthii }\end{array}$ & $\begin{array}{c}3.4 \pm \\
0.9\end{array}$ & $\begin{array}{l}2410 \\
\pm 1.8\end{array}$ & $\begin{array}{c}800 \\
\pm \\
2.8\end{array}$ & $\begin{array}{c}170926 . \\
3 \pm 4.0\end{array}$ & $\begin{array}{l}501 \\
0 \pm \\
2.6\end{array}$ & $\begin{array}{l}2290.3 \\
\pm 7.10\end{array}$ & $\begin{array}{c}177.32 \\
\pm 0.4\end{array}$ & $\begin{array}{c}85.25 \pm \\
3.4\end{array}$ & $\begin{array}{c}11.92 \pm \\
4.1\end{array}$ & 0.01 \\
\hline M. staudtii & $\begin{array}{c}16 \pm \\
2.5\end{array}$ & $\begin{array}{l}1100 \\
\pm 6.1\end{array}$ & $\begin{array}{c}102 \\
0 \\
\pm 2.1\end{array}$ & $\begin{array}{c}138173 \\
8 \pm 1.1\end{array}$ & $\begin{array}{l}291 \\
0 \pm \\
0.1\end{array}$ & $\begin{array}{c}2401.1 \\
\pm 4.0\end{array}$ & $\begin{array}{l}184.47 \\
\pm 0,12\end{array}$ & $\begin{array}{c}132.99 \\
\pm 3.0\end{array}$ & $\begin{array}{c}15.00 \pm \\
3.1\end{array}$ & 0.17 \\
\hline P. ambigua & $\begin{array}{c}6.1 \pm \\
0.6\end{array}$ & $\begin{array}{l}2220 \\
\pm 2.1\end{array}$ & $\begin{array}{c}220 \\
\pm \\
3.5\end{array}$ & $\begin{array}{c}4355.54 \\
\pm 6.0\end{array}$ & $\begin{array}{l}139 \\
0 \pm \\
1.0\end{array}$ & $\begin{array}{l}10289 \\
6 \pm 1.3\end{array}$ & $\begin{array}{c}804.30 \\
\pm 2.7\end{array}$ & $\begin{array}{c}338.96 \\
\pm 4.6\end{array}$ & $\begin{array}{c}40.17 \pm \\
2.0\end{array}$ & 2.36 \\
\hline P. longifolia & $\begin{array}{c}1.4 \pm \\
2.1\end{array}$ & $\begin{array}{l}1400 \\
\pm 3.1\end{array}$ & $\begin{array}{l}146 \\
0 \pm \\
2.3 \\
\end{array}$ & $\begin{array}{c}173791 \\
7 \pm 1.4\end{array}$ & $\begin{array}{l}172 \\
0 \pm \\
2.0 \\
\end{array}$ & $\begin{array}{c}7220.1 \\
\pm 1.0\end{array}$ & $\begin{array}{c}174.15 \\
\pm 1.9\end{array}$ & $\begin{array}{c}161.25 \\
\pm 3.4\end{array}$ & $\begin{array}{c}15.03 \pm \\
2.4\end{array}$ & 0.04 \\
\hline $\begin{array}{l}P . \\
\text { macrophylla }\end{array}$ & $\begin{array}{c}0.5 \pm \\
1.1\end{array}$ & $\begin{array}{l}2310 \\
\pm 2.4\end{array}$ & $\begin{array}{l}347 \\
0 \pm \\
3.4\end{array}$ & $\begin{array}{c}220424 \\
6 \pm 3.1\end{array}$ & $\begin{array}{l}711 \\
0 \pm \\
3.1\end{array}$ & $\begin{array}{c}3659.7 \\
\pm 1.5\end{array}$ & $\begin{array}{c}689.63 \\
\pm 3.2\end{array}$ & $\begin{array}{c}355.54 \\
\pm 2.2\end{array}$ & $\begin{array}{c}21.43 \pm \\
3.3\end{array}$ & 0.02 \\
\hline R. corymbosa & $\begin{array}{c}41 \pm \\
1.0\end{array}$ & $\begin{array}{l}1260 \\
\pm 0.4\end{array}$ & $\begin{array}{l}151 \\
0 \pm \\
0.5\end{array}$ & $\begin{array}{c}31331.3 \\
\pm 2.7\end{array}$ & $\begin{array}{l}282 \\
0 \pm \\
1.0\end{array}$ & $\begin{array}{c}5541.0 \\
\pm 3.6\end{array}$ & $\begin{array}{c}105.98 \\
\pm 0.8\end{array}$ & $\begin{array}{c}16.20 \pm \\
6.4\end{array}$ & $\begin{array}{c}188.81 \pm \\
3.0\end{array}$ & 0.18 \\
\hline R. heudelotii & $\begin{array}{c}2.1 \pm \\
2.4\end{array}$ & $\begin{array}{l}2780 \\
\pm 3.8\end{array}$ & $\begin{array}{c}210 \\
\pm \\
8.1 \\
\end{array}$ & $\begin{array}{c}197425 . \\
0 \pm 4.0\end{array}$ & $\begin{array}{l}139 \\
0 \pm \\
3.2 \\
\end{array}$ & $\begin{array}{c}2966.4 \\
\pm 5.0\end{array}$ & $\begin{array}{c}302.10 \\
\pm 2.1\end{array}$ & $\begin{array}{c}234.53 \\
\pm 5.4\end{array}$ & $\begin{array}{c}27.80 \pm \\
5.2\end{array}$ & 0.02 \\
\hline $\begin{array}{l}P . \\
\text { scrobiculatum }\end{array}$ & $\begin{array}{l}5.1 \pm \\
0.4\end{array}$ & $\begin{array}{l}1040 \\
\pm 1.8\end{array}$ & $\begin{array}{c}640 \\
\pm \\
1.0\end{array}$ & $\begin{array}{c}140533 \\
3 \pm 3.2\end{array}$ & $\begin{array}{l}130 \\
0 \pm \\
0.2\end{array}$ & $\begin{array}{l}58483 \\
6 \pm 0.4\end{array}$ & $\begin{array}{c}912.64 \\
\pm 2.3\end{array}$ & $\begin{array}{c}248.00 \\
\pm 2.1\end{array}$ & $\begin{array}{c}4855.94 \\
\pm 1.0\end{array}$ & 0.42 \\
\hline $\begin{array}{l}T . \\
\text { mauritianum }\end{array}$ & $\begin{array}{c}11 \pm \\
4.4\end{array}$ & $\begin{array}{c}990 \pm \\
1,6\end{array}$ & $\begin{array}{c}790 \\
\pm \\
3.8\end{array}$ & $\begin{array}{c}53120.0 \\
\pm 3.3\end{array}$ & $\begin{array}{l}147 \\
0 \pm \\
4.2\end{array}$ & $\begin{array}{l}10406 . \\
0 \pm 5.0\end{array}$ & $\begin{array}{c}224.10 \\
\pm 1.0\end{array}$ & $\begin{array}{c}157.7 \pm \\
2.9\end{array}$ & $\begin{array}{c}2089.51 \\
\pm 2.8\end{array}$ & 0.20 \\
\hline U. guineensis & $\begin{array}{c}0.9 \pm \\
3.4\end{array}$ & $\begin{array}{l}1170 \\
\pm 3,9\end{array}$ & $\begin{array}{c}220 \\
\pm \\
6.8\end{array}$ & $\begin{array}{c}203850 . \\
8 \pm 4.0\end{array}$ & $\begin{array}{l}122 \\
0 \pm \\
0.2\end{array}$ & $\begin{array}{l}10418 \\
7 \pm 3.0\end{array}$ & $\begin{array}{c}267.1 \pm \\
2.4\end{array}$ & $\begin{array}{c}224.6 \pm \\
1.5\end{array}$ & $\begin{array}{c}42.45 \pm \\
6.1\end{array}$ & 0.05 \\
\hline
\end{tabular}

Chemical composition of some plant parts used by mandrill

The phytochemical screening results of some plants from Mandrillus sphinx diet are presented in the Table 3 . The phytochemical result certifies the abundance of several secondary metabolites such as polyphenols, tannins, alkaloids, triterpenoids compounds and reduced sugar in different extracts.

Table 3:Chemical composition of some plant parts from Mandrill population diet.

\begin{tabular}{|l|c|c|c|c|c|c|c|c|c|}
\hline \multirow{2}{*}{ Espèces végétales } & \multicolumn{9}{|c|}{ Secondarymetabolites } \\
\cline { 2 - 10 } & Tan & Sap & Alca & Poly & Flav & S/Red & T/St. & H/Car & Anth \\
\hline A. cf stipulatum & - & +++ & - & +++ & +++ & +++ & ++ & - & - \\
\hline
\end{tabular}




\begin{tabular}{|c|c|c|c|c|c|c|c|c|c|}
\hline A. daniellii & +++ & ++ & + & ++ & +++ & +++ & ++ & - & + \\
\hline Aframomum sp & - & +++ & + & +++ & +++ & +++ & +++ & - & + \\
\hline A. pentagyna & +++ & ++ & +++ & +++ & +++ & +++ & + & - & - \\
\hline A. rubiginosa & +++ & + & +++ & +++ & +++ & + & + & + & - \\
\hline A. floribunda & +++ & - & - & +++ & +++ & + & ++ & - & - \\
\hline A. africanum & +++ & +++ & - & +++ & +++ & + & ++ & - & - \\
\hline B. fistulosa & - & ++ & +++ & +++ & +++ & - & - & - & ++ \\
\hline B. bracteosa & + & - & +++ & ++ & +++ & - & - & + & - \\
\hline B. ferruginea & +++ & +++ & ++ & +++ & +++ & - & +++ & - & - \\
\hline L. welwitschii & +++ & ++ & + & +++ & +++ & +++ & +++ & - & + \\
\hline Landolphia sp & - & ++ & +++ & - & +++ & +++ & +++ & - & - \\
\hline C. corniculata & +++ & +++ & +++ & +++ & +++ & +++ & +++ & - & +++ \\
\hline C. mucunoides & ++ & +++ & +++ & +++ & +++ & + & - & - & ++ \\
\hline C. cerinanthum & - & + & + & ++ & ++ & +++ & ++ & - & - \\
\hline C. sylvaticus & + & ++ & ++ & +++ & +++ & +++ & +++ & - & - \\
\hline D. dinklagei & +++ & - & ++ & +++ & +++ & +++ & +++ & - & - \\
\hline D. corbisieri & +++ & - & +++ & - & +++ & - & +++ & - & - \\
\hline D. tessmannii & - & - & ++ & +++ & +++ & - & - & - & - \\
\hline D. africana & +++ & +++ & +++ & +++ & +++ & +++ & +++ & - & - \\
\hline D. cf umbellatum & +++ & - & ++ & ++ & +++ & +++ & +++ & - & + \\
\hline D. multiflora & - & - & +++ & +++ & +++ & - & - & - & - \\
\hline E. glomerata & +++ & ++ & - & +++ & +++ & +++ & +++ & - & ++ \\
\hline Haumania sp & + & - & ++ & +++ & +++ & ++ & - & - & - \\
\hline H. gabunense & +++ & ++ & ++ & +++ & +++ & +++ & +++ & - & +++ \\
\hline J. gabonensis & + & +++ & +++ & ++ & +++ & +++ & ++ & - & - \\
\hline L. glabra & - & ++ & ++ & +++ & +++ & +++ & ++ & - & - \\
\hline Landolphia sp & +++ & - & +++ & +++ & +++ & +++ & +++ & - & +++ \\
\hline L. batangensis & - & - & - & +++ & ++ & +++ & ++ & - & - \\
\hline
\end{tabular}

Tan: tannins, T/St: triterpenoids and sterols, Sap: saponins, Alca: alkaloids, Poly: polyphenols, Flav: flavonoids, S/Red: reduced sugar, Anth: anthocyanins, HCar: cardiotonic heterosids, +++: very abundant, ++: Abundant, +: lower, -: negatif test.

Total phenolic, tannins and flavonoids content.

The table 4 showed the polyphenols content in some food plants from mandrill diet. Levels of phenolic content were expressed in terms of gallic acid equivalent (GAE). The results showed that total phenols compounds content had ranged between $0.06 \pm 1.18$ and $44.46 \pm 0.10 \mathrm{~g}$ EAG/100g of dried matter (DM). Antinutritional compounds values (tannins) varies between $0.005 \pm 2.1$ and $28.15 \pm 0.22 \mathrm{~g}$ EAT/100g DM and flavonoids content vary between $0.021 \pm 0.29$ and $11.45 \pm 0.03 \mathrm{~g} \mathrm{EQ} / 100 \mathrm{~g} \mathrm{DM}$

The highest content of phenolic compounds was obtained with fruits of Renealmia macrocolea $(44.46 \pm 0.10 \mathrm{~g}$ EAG/100g DM), Haumania liebrechtsiana (38.80 $\pm 0.08 \mathrm{~g}$ EAG/100g de DM) and Aframomum alboviolaceum $(35.76 \pm 0.03 \mathrm{~g} \mathrm{EAG} / 100 \mathrm{~g} \mathrm{DM})$.

Thehighest values tannins compounds were unregistered with fruits Haumania liebrechtsiana $(28.15 \pm 0.22 \mathrm{~g}$ EAT/100g DM), Aframomum alboviolaceum $(16.67 \pm 0.91 \mathrm{~g}$ EAT/100g DM) and stems of Manniophyton fulvum $(11.21 \pm 0.57 \mathrm{~g} \mathrm{EAT} / 100 \mathrm{~g} \mathrm{DM})$.

Table 4:-Total phenolic compounds content of some food plants used by mandrills. 


\begin{tabular}{|c|c|c|c|c|}
\hline & parts & (g EAG/100 g) & (g EAT/100 g) & (g EQ/100 g) \\
\hline Aframomum alboviolaceum & Fruits & $35.76 \pm 0.03$ & $16.67 \pm 0.91$ & $2.75 \pm 0.03$ \\
\hline Aframomum cf polyanthum & Fruits & $74.01 \pm 0.01$ & $1.62 \pm 0.03$ & $2.33 \pm 0.08$ \\
\hline Aframomum daniellii & Fruits & $8.99 \pm 0.03$ & $12.78 \pm 1.23$ & $1.78 \pm 0.23$ \\
\hline Aframomum sp & Fruits & $18.64 \pm 0.04$ & $0.96 \pm 0.90$ & $6.61 \pm 1.20$ \\
\hline Alchornea floribunda & Fruits & $10.86 \pm 1.3$ & $1.85 \pm 2.12$ & $2.09 \pm 0.50$ \\
\hline Antrocaryon klaineanum & Fruits & $4.46 \pm 0.10$ & $3.99 \pm 0.01$ & $5.94 \pm 0.15$ \\
\hline Barteria bracteosa & Fruits & $6.15 \pm 3.10$ & $0.08 \pm 6.12$ & $0.14 \pm 0.20$ \\
\hline Colopogonium mucunoides & Fruits & $4.15 \pm 0.70$ & $0.11 \pm 0.01$ & $0.53 \pm 0.04$ \\
\hline Crotons sylvaticus & Fruits & $12.74 \pm 0.22$ & $6.56 \pm 0.46$ & $9.38 \pm 0.08$ \\
\hline Elaeis guineensis & fruits & $1.01 \pm 1.25$ & $0.01 \pm 0.65$ & $3.94 \pm 0.03$ \\
\hline Eriosema glomerata & Fruits & $3.69 \pm 0.01$ & $2.27 \pm 0.08$ & $0.05 \pm 1.22$ \\
\hline Geophila afzelii & Fruits & $1.40 \pm 0.11$ & $0.95 \pm 0.02$ & $0.59 \pm 0.05$ \\
\hline Haumania liebrechtsiana & Seeds & $38,80 \pm 0.08$ & $28.15 \pm 0.22$ & $0.44 \pm 0.01$ \\
\hline Hylodendron gabunense & Seeds & $3.18 \pm 1.02$ & $1.97 \pm 3.12$ & $0.76 \pm 0.01$ \\
\hline Landolphia hirsuta & Fruits & $7.40 \pm 0.11$ & $0.43 \pm 0.30$ & $0.025 \pm 0.02$ \\
\hline Lannea welwitschii & Fruits & $0.69 \pm 0.90$ & $0.13 \pm 6.30$ & $0.04 \pm 1.20$ \\
\hline Lasianthus batangensis & Fruits & $5.76 \pm 0.25$ & $2.91 \pm 1.05$ & $1.25 \pm 0.01$ \\
\hline Macaranga schweinfurthii & Petioles & $17.34 \pm 0.10$ & $4.68 \pm 0.12$ & $1.11 \pm 0.01$ \\
\hline Maesobotrya klaineana & Fruits & $0.28 \pm 3.10$ & $0.05 \pm 0.81$ & $0.09 \pm 0.01$ \\
\hline Manniophyton fulvum & Stems & $22.75 \pm 0.32$ & $11.21 \pm 0.57$ & $6.69 \pm 0.01$ \\
\hline Medelina mirabilis & Fruits & $3.03 \pm 0.05$ & $1.98 \pm 1.01$ & $0.38 \pm 0.02$ \\
\hline Megaphrynium macrostachum & Fruits & $4.01 \pm 0.01$ & $1.96 \pm 0.11$ & $1.62 \pm 0.03$ \\
\hline Microdesmis haumaniana & Seeds & $0.98 \pm 2.31$ & $0.19 \pm 0.01$ & $0.07 \pm 6.20$ \\
\hline Musanga cecropioides & Fruits & $18.53 \pm 0.09$ & $2.41 \pm 1.5$ & $3.36 \pm 0.02$ \\
\hline Nymphaea maculata & Leaves & $14.93 \pm 1.0$ & $8.19 \pm 0.77$ & $11.45 \pm 0.03$ \\
\hline Palisota ambigua & Fruits & $16.35 \pm 0.09$ & $9.03 \pm 0.3$ & $2.44 \pm 0.03$ \\
\hline Paspalum scrobiculatum & Seeds & $1.49 \pm 0.41$ & $0.12 \pm 0.03$ & $0.11 \pm 2.17$ \\
\hline Pentaclethra eetveldeana & Flowers & $9.97 \pm 0.07$ & $1.52 \pm 0.29$ & $6.08 \pm 0.04$ \\
\hline Pentaclethra macrophylla & Seeds & $6.37 \pm 0.29$ & $2.11 \pm 0.21$ & $0.025 \pm 0.02$ \\
\hline Plagiostyles africana & Seeds & $2.04 \pm 1.20$ & $0.26 \pm 1.04$ & $0.67 \pm 1.01$ \\
\hline Renealmia macrocolea & Fruits & $44.46 \pm 0.10$ & $10.69 \pm 0.79$ & $0.44 \pm 0.01$ \\
\hline Rhynchospora corymbosa & Fruits & $3.66 \pm 0.02$ & $2.26 \pm 0.03$ & $0.62 \pm 0.07$ \\
\hline Ricinodendron heudelotii & Seeds & $3.01 \pm 0.20$ & $0.01 \pm 0.49$ & $0.021 \pm 0.15$ \\
\hline Sarcophrynium brachystachyum & Stems & $1.31 \pm 0.03$ & $0.11 \pm 0.08$ & $1.96 \pm 0.10$ \\
\hline Smilax anceps & Fruits & $6.93 \pm 0.05$ & $5.65 \pm 0.82$ & $3.31 \pm 0.91$ \\
\hline Trachyphrynium braunianum & Stems & $0.66 \pm 0.24$ & $0.02 \pm 2.21$ & $0.04 \pm 3.20$ \\
\hline Tricalysia cf. breteleri & Fruits & $4.45 \pm 0.13$ & $3.09 \pm 0.78$ & $0.021 \pm 0.29$ \\
\hline Tristemma mauritianum & Leaves & $28.45 \pm 1.2$ & $3.21 \pm 3.04$ & $3.07 \pm 0.40$ \\
\hline Uapaca guineensis & Fruits & $0.06 \pm 1.18$ & $0.005 \pm 2.1$ & $0.04 \pm 0.01$ \\
\hline Uvaria scrabida & Fruits & $0.65 \pm 0.17$ & $0.07 \pm 0.24$ & $0.12 \pm 2.13$ \\
\hline
\end{tabular}

\section{Antioxidant Activity Index (AAI)}

The results of the antioxidant activity of some fruits and seeds extracts of food plants from mandrill diet and consumed by local population are presented in the table 5 . 
The AAI of the extracts were ranged between $0.34 \pm 2.7$ and $5.19 \pm 3.0$. The highest AAI values have been obtained with fruits of two Aframomum species (A. cf polyanthum (5.19 \pm 3.0 ) and A. alboviolaceum (4.16 \pm 0.2$)$ ), fruits of Pseudospondias longifolia (4.02 \pm 2.5$)$, Chrysophyllum lacourtianum $(3.50 \pm 2.6)$ and Landolphia mannii (3.08 \pm $0.0)$ and can be compared to AAI of Vitamin C (10.22 \pm 0.9$)$ and BHA (3.54 \pm 6.93$)$. The lowest values have recorded with fruits of Antrocaryon klaineanum, Uapaca guineensis and Pentaclethra macrophylla $(0.34 \pm 2.7,0.56$ \pm 1.1 and $0.81 \pm 0.3$ resp.).

Table 5:-Antioxidant activities some fruits and seeds consumed by mandrills and local population.

\begin{tabular}{|l|l|c|c|c|c|}
\hline Vegetables species & Parties & Equations & $\mathbf{R}^{2}$ & $\mathbf{I C}_{\mathbf{5 0}}(\boldsymbol{\mu g} / \mathbf{~ m L})$ & IAA \\
\hline Aframomum alboviolaceum & Fruits & $\mathrm{Y}=1.813 \mathrm{X}+3.639$ & 0.936 & $12.01 \pm 1.2$ & $4.16 \pm 0.2$ \\
\hline Aframomum cf polyanthum & Fruits & $\mathrm{Y}=1.314 \mathrm{X}+06.91$ & 0.967 & $9.64 \pm 2.4$ & $5.19 \pm 3.0$ \\
\hline Aframomum daniellii & Fruits & $\mathrm{Y}=1.813 \mathrm{X}+20.73$ & 0.893 & $19.10 \pm 0.1$ & $2.61 \pm 2.0$ \\
\hline Aframomum sp & Fruits & $\mathrm{Y}=1.946 \mathrm{X}+17.21$ & 0.893 & $16.85 \pm 2.1$ & $2.97 \pm 3.8$ \\
\hline Antrocaryon klaineanum & Fruits & $\mathrm{Y}=1.946 \mathrm{X}+17.21$ & 0.998 & $147.1 \pm 1.0$ & $0.34 \pm 2.7$ \\
\hline Chrysophyllum africanum & Fruits & $\mathrm{Y}=1.691 \mathrm{X}+21.82$ & 0.922 & $39.85 \pm 3.7$ & $2.06 \pm 2.0$ \\
\hline Chrysophyllum lacourtianum & Fruits & $\mathrm{Y}=1.275 \mathrm{X}+02.63$ & 0.996 & $37.16 \pm 0.1$ & $3.50 \pm 2.6$ \\
\hline Landolphia mannii & Fruits & $\mathrm{Y}=0.466 \mathrm{X}+0.992$ & 0.991 & $16.21 \pm 0.4$ & $3.08 \pm 0.0$ \\
\hline Pentaclethra macrophylla & Seeds & $\mathrm{Y}=1.894 \mathrm{X}+13.61$ & 0.936 & $61.29 \pm 2.7$ & $0.81 \pm 0.3$ \\
\hline Pseudospondias longifolia & Fruits & $\mathrm{Y}=1.739 \mathrm{X}+24.51$ & 0.997 & $12.42 \pm 3.2$ & $4.02 \pm 2.5$ \\
\hline Uapaca guineensis & Fruits & $\mathrm{Y}=1.813 \mathrm{X}+20.73$ & 0.863 & $89.52 \pm 0.1$ & $0.56 \pm 1.1$ \\
\hline Vitamine C & & $\mathrm{Y}=5.120 \mathrm{X}+01.46$ & 0.971 & $4.89 \pm 2.80$ & $10.22 \pm 0.9$ \\
\hline BHA & $\mathrm{Y}=3.621 \mathrm{X}+0.330$ & 0.962 & $7.21 \pm 0.90$ & $3.54 \pm 6.93$ \\
\hline
\end{tabular}

Inhibition zone diameters by disc assay of some food plants from mandrill diet used in traditional medicine by local population.

The inhibition zone diameters (IZD) obtained in the antimicrobial susceptibility assays for the plants extracts tested and standard antimicrobial drug discs are showed in Table 6.

The results showed that IZD recorded were ranged between 7 and $22 \mathrm{~mm}$. The highest IZD was observed with the fruits of Eriosema glomerata $(22 \mathrm{~mm})$ and leaves of Piptadeniastrum africanum $(20 \mathrm{~mm})$. The lowest IZD have been obtained with seeds Xylopia staudtii $(7 \mathrm{~mm})$, leaves of Tristemma mauritianum $(8 \mathrm{~mm})$. These results showed also that some plant parts of some plants such as Medelina mirabilis, Pentaclethra eetveldeana and Eriosema glomerata have been active on the strains of Escherichia coli and Enterobacter cloacae complex resistant at standard antibiotic.

Table 6:-Zone inhibition diameters of some plant parts from mandrill diet.

\begin{tabular}{|l|c|c|c|c|c|c|c|c|}
\hline \multirow{2}{*}{ Plant species } & \multicolumn{7}{|c|}{ Zone inhibition diameters (mm) } \\
\cline { 2 - 10 } & EC121 & CY127 & EC129 & KPP32 & EC292 & ECC110 & EC114 & PA383 \\
\hline M. mirabilis & 17 & - & - & 14 & 13 & 14 & 11 & 15 \\
\hline P. eetveldeana & 15 & 12 & 19 & - & 16 & 15 & - & 14 \\
\hline P. africanum & - & 13 & - & - & 9 & 20 & - & 20 \\
\hline A. floribunda & 9 & 11 & 17 & 12 & - & - & 19 & 13 \\
\hline T. mauritianum & 12 & 9 & 13 & 13 & 9 & 14 & 8 & 10 \\
\hline M. staudtii & - & - & 15 & 15 & - & - & 7 & - \\
\hline M. haumaniana & - & 12 & 22 & 10 & - & - & - & - \\
\hline E. glomerata & 18 & 13 & - & 22 & 12 & 11 & 10 & 8 \\
\hline Haumania sp & - & 7 & - & 17 & 11 & - & - & - \\
\hline ERTA & - & 12 & - & 19 & - & - & 13 & 17 \\
\hline
\end{tabular}

EC: Escherichia coli, CY: Citrobacter youngae, PA: Pseudomonas aeruginosa, KPP: Klebsiella pneumoniae ssp pneumoniae, ECC: Enterobacter cloacae complex: -: negative test; ERTA: Ertapenem. 
The table 7 presented the minimum inhibitory concentration (MIC) and minimum bactericidal concentration (MBC) values confirmed the antibacterial activity of some food plants from mandrill diet.

The lowest MIC $(<0.19 \mathrm{mg} / \mathrm{mL})$ was recorded with the leaves of Tristemma mauritianum and fruits of Microdesmis haumaniana. Other parts of plant such as fruits of Medelina mirabilis and Alchornea floribunda have also showed the lower minimum inhibitory concentration $(0.39$ and $0.78 \mathrm{mg} / \mathrm{ml}$ respectively).

The lowest MBC $(1.56 \mathrm{mg} / \mathrm{mL})$ was obtained with fruits and leaves extracts of Alchornea floribunda and Tristemma mauritianum respectively.

Table 7:-Minimum inhibitory concentration (MIC) and minimum concentration bactericidal of some plants from mandrill diet and used in traditional medicine.

\begin{tabular}{|c|c|c|c|c|c|c|c|c|c|c|c|c|c|c|c|c|}
\hline \multirow{3}{*}{$\begin{array}{l}\text { Plant } \\
\text { species }\end{array}$} & \multicolumn{16}{|c|}{$\mathrm{MCl}$ and $\mathrm{MCB}(\mathrm{mg} / \mathrm{ml})$} \\
\hline & \multicolumn{2}{|c|}{ EC121 } & \multicolumn{2}{|c|}{ CY127 } & \multicolumn{2}{|c|}{ EC129 } & \multicolumn{2}{|c|}{ KPP32 } & \multicolumn{2}{|c|}{ EC292 } & \multicolumn{2}{|c|}{ ECC110 } & \multicolumn{2}{|c|}{ EC114 } & \multicolumn{2}{|c|}{ PA383 } \\
\hline & $\begin{array}{c}\text { MC } \\
\text { I }\end{array}$ & $\begin{array}{l}M \\
\text { CB }\end{array}$ & $\begin{array}{c}\text { MC } \\
\text { I }\end{array}$ & $\begin{array}{c}\text { MC } \\
\text { B }\end{array}$ & $\begin{array}{l}\mathrm{M} \\
\mathrm{Cl}\end{array}$ & $\begin{array}{c}\text { MC } \\
\text { B }\end{array}$ & $\begin{array}{c}\text { MC } \\
\text { I }\end{array}$ & $\begin{array}{c}\text { MC } \\
\text { B }\end{array}$ & $\begin{array}{l}\mathrm{M} \\
\mathrm{Cl}\end{array}$ & $\begin{array}{c}\text { MC } \\
\text { B }\end{array}$ & $\begin{array}{l}\mathrm{M} \\
\mathrm{Cl}\end{array}$ & $\begin{array}{c}\text { MC } \\
\text { B }\end{array}$ & $\begin{array}{l}\mathrm{M} \\
\mathrm{Cl}\end{array}$ & $\begin{array}{c}\text { MC } \\
\text { B }\end{array}$ & $\begin{array}{l}\mathrm{M} \\
\mathrm{Cl}\end{array}$ & $\begin{array}{c}\text { MC } \\
\text { B }\end{array}$ \\
\hline $\begin{array}{l}\text { M. } \\
\text { mirabilis }\end{array}$ & $\begin{array}{c}0.7 \\
8\end{array}$ & 50 & $\mathrm{Nd}$ & $\mathrm{Nd}$ & $\mathrm{Nd}$ & $\mathrm{Nd}$ & $\begin{array}{c}2.5 \\
0\end{array}$ & $\begin{array}{c}10 \\
0\end{array}$ & $\begin{array}{c}12 \\
.5\end{array}$ & $\begin{array}{l}>2 \\
00\end{array}$ & $\begin{array}{l}6 . \\
25\end{array}$ & $\begin{array}{l}>2 \\
00\end{array}$ & $\begin{array}{l}3 . \\
13\end{array}$ & 25 & $\begin{array}{c}12 \\
.5\end{array}$ & 50 \\
\hline $\begin{array}{l}P . \\
\text { eetveldean } \\
a\end{array}$ & $\begin{array}{c}6.2 \\
5\end{array}$ & $\begin{array}{c}10 \\
0\end{array}$ & $\begin{array}{c}12 . \\
5\end{array}$ & $\begin{array}{l}>2 \\
00\end{array}$ & $\begin{array}{l}6 . \\
25\end{array}$ & $\begin{array}{l}>2 \\
00\end{array}$ & $\mathrm{Nd}$ & $\mathrm{Nd}$ & 50 & $\begin{array}{c}20 \\
0\end{array}$ & $\begin{array}{c}12 \\
.5\end{array}$ & $\begin{array}{l}>2 \\
00\end{array}$ & $\mathrm{Nd}$ & $\mathrm{Nd}$ & $\begin{array}{l}6 . \\
25\end{array}$ & $\begin{array}{l}>2 \\
00\end{array}$ \\
\hline $\begin{array}{l}P . \\
\text { africanum }\end{array}$ & $\mathrm{Nd}$ & $\mathrm{Nd}$ & 25 & $\begin{array}{c}20 \\
0\end{array}$ & $\mathrm{Nd}$ & $\mathrm{Nd}$ & $\mathrm{Nd}$ & $\mathrm{Nd}$ & $\begin{array}{l}6 . \\
25\end{array}$ & $\begin{array}{c}20 \\
0\end{array}$ & $\begin{array}{l}3 . \\
13\end{array}$ & $\begin{array}{l}>2 \\
00\end{array}$ & $\mathrm{Nd}$ & $\mathrm{Nd}$ & $\begin{array}{l}3 . \\
13\end{array}$ & 25 \\
\hline $\begin{array}{l}\text { A. } \\
\text { floribunda }\end{array}$ & $\begin{array}{c}0.3 \\
9\end{array}$ & 25 & $\begin{array}{c}1.5 \\
6\end{array}$ & 50 & $\begin{array}{l}3 . \\
13\end{array}$ & $\begin{array}{l}>2 \\
00\end{array}$ & $\begin{array}{c}0.7 \\
8\end{array}$ & $\begin{array}{c}10 \\
0\end{array}$ & $\mathrm{Nd}$ & $\mathrm{Nd}$ & $\mathrm{Nd}$ & $\mathrm{Nd}$ & $\begin{array}{l}3 . \\
13\end{array}$ & 25 & $\begin{array}{l}6 . \\
25 \\
\end{array}$ & 25 \\
\hline $\begin{array}{l}T . \\
\text { mauritianu } \\
m\end{array}$ & $\begin{array}{l}<0 . \\
19\end{array}$ & 25 & $\begin{array}{l}<0 . \\
19\end{array}$ & 25 & $\begin{array}{c}12 \\
.5\end{array}$ & $\begin{array}{c}10 \\
0\end{array}$ & $\begin{array}{l}<0 . \\
15\end{array}$ & $\mathrm{Nd}$ & $\begin{array}{l}6 . \\
25\end{array}$ & $\begin{array}{l}>2 \\
00\end{array}$ & $\begin{array}{l}0 . \\
78\end{array}$ & 25 & $\begin{array}{l}6 . \\
25\end{array}$ & $\begin{array}{c}12 . \\
5\end{array}$ & $\begin{array}{l}3 . \\
13\end{array}$ & $\begin{array}{l}>2 \\
00\end{array}$ \\
\hline M. staudtii & $\mathrm{Nd}$ & $\mathrm{Nd}$ & $\mathrm{Nd}$ & $\mathrm{Nd}$ & 25 & $\begin{array}{l}>2 \\
00\end{array}$ & $\begin{array}{c}12 . \\
5\end{array}$ & 50 & $\mathrm{Nd}$ & $\mathrm{Nd}$ & $\mathrm{Nd}$ & 25 & $\begin{array}{l}12 \\
.5\end{array}$ & $\begin{array}{c}10 \\
0\end{array}$ & $\mathrm{Nd}$ & $\mathrm{Nd}$ \\
\hline $\begin{array}{l}\text { M. } \\
\text { haumania } \\
\text { na }\end{array}$ & $\mathrm{Nd}$ & $\mathrm{Nd}$ & $\begin{array}{l}<0 . \\
19\end{array}$ & 25 & $\begin{array}{l}1 . \\
56\end{array}$ & 50 & 25 & $\begin{array}{l}>2 \\
00\end{array}$ & $\mathrm{Nd}$ & $\mathrm{Nd}$ & $\mathrm{Nd}$ & $\mathrm{Nd}$ & $\mathrm{Nd}$ & $\mathrm{Nd}$ & $\mathrm{Nd}$ & $\mathrm{Nd}$ \\
\hline $\begin{array}{l}\text { E. } \\
\text { glomerata }\end{array}$ & $\begin{array}{c}3.1 \\
3\end{array}$ & 50 & 50 & $\begin{array}{l}>2 \\
00\end{array}$ & $\mathrm{Nd}$ & $\mathrm{Nd}$ & $\begin{array}{c}1.5 \\
6\end{array}$ & 25 & 25 & $\begin{array}{l}>2 \\
00\end{array}$ & $\begin{array}{l}3 . \\
13\end{array}$ & $\begin{array}{c}10 \\
0\end{array}$ & $\begin{array}{c}10 \\
0\end{array}$ & $\begin{array}{l}>2 \\
00\end{array}$ & $\begin{array}{l}6 . \\
25 \\
\end{array}$ & $\begin{array}{c}10 \\
0\end{array}$ \\
\hline $\begin{array}{l}\text { Haumania } \\
\text { sp }\end{array}$ & $\mathrm{Nd}$ & $\mathrm{Nd}$ & 100 & $\begin{array}{l}>2 \\
00\end{array}$ & $\mathrm{Nd}$ & $\mathrm{Nd}$ & 100 & $\begin{array}{l}>2 \\
00\end{array}$ & 50 & $\begin{array}{l}>2 \\
00\end{array}$ & $\mathrm{Nd}$ & $\mathrm{Nd}$ & $\mathrm{Nd}$ & $\mathrm{Nd}$ & $\mathrm{Nd}$ & $\mathrm{Nd}$ \\
\hline ERTA & $\mathrm{Nd}$ & $\mathrm{Nd}$ & $\begin{array}{c}1.5 \\
6\end{array}$ & $\begin{array}{c}12 . \\
3\end{array}$ & $\mathrm{Nd}$ & $\mathrm{Nd}$ & $\begin{array}{c}3.1 \\
3\end{array}$ & $\begin{array}{c}6.2 \\
5\end{array}$ & $\mathrm{Nd}$ & $\mathrm{Nd}$ & $\mathrm{Nd}$ & $\mathrm{Nd}$ & $\begin{array}{l}1 . \\
56\end{array}$ & $\begin{array}{c}12 . \\
5\end{array}$ & $\begin{array}{l}1 . \\
56\end{array}$ & $\begin{array}{c}3.2 \\
5\end{array}$ \\
\hline
\end{tabular}

$\mathrm{Nd}=$ not determinated

The results of report between MBC/MIC are presented in the table 8 . These results showed that the values of this report were recorded between 2 and 64. The lowest values were obtained with Tristemma mauritianum (MBC/MIC $=2$ ), Medelina mirabilis, Pentaclethra eetveldeana and Alchornea floribunda (MBC/MIC $=4$ ). These lowest MBC/MIC were obtained on the bacterial strains of Escherichia coli and Pseudomonas aeruginosa.

Table 8:-The report MBC/MIC of plant part extracts analyzed.

\begin{tabular}{|l|c|c|c|c|c|c|c|c|}
\hline \multirow{2}{*}{ Vegetables species } & \multicolumn{10}{|c|}{ MBC/MIC } \\
\cline { 2 - 9 } & EC121 & CY127 & EC129 & KPP32 & EC292 & ECC110 & EC114 & PA383 \\
\hline Medelina mirabilis & 64 & $\mathrm{Nd}$ & $\mathrm{Nd}$ & 40 & $\mathrm{Nd}$ & $\mathrm{Nd}$ & 8 & 4 \\
\hline
\end{tabular}




\begin{tabular}{|l|c|c|c|c|c|c|c|c|}
\hline Pentaclethra eetveldeana & 16 & $\mathrm{Nd}$ & $\mathrm{Nd}$ & $\mathrm{Nd}$ & 4 & $\mathrm{Nd}$ & $\mathrm{Nd}$ & $\mathrm{Nd}$ \\
\hline Piptadeniastrum africanum & $\mathrm{Nd}$ & 8 & $\mathrm{Nd}$ & $\mathrm{Nd}$ & 32 & $\mathrm{Nd}$ & $\mathrm{Nd}$ & 8 \\
\hline Alchornea floribunda & 64 & 32 & $\mathrm{Nd}$ & $\mathrm{Nd}$ & $\mathrm{Nd}$ & $\mathrm{Nd}$ & 8 & 4 \\
\hline Tristemma mauritianum & $\mathrm{Nd}$ & $\mathrm{Nd}$ & 8 & $\mathrm{Nd}$ & $\mathrm{Nd}$ & 32.1 & 2 & $\mathrm{Nd}$ \\
\hline Maesobotrya staudtii & $\mathrm{Nd}$ & $\mathrm{Nd}$ & $\mathrm{Nd}$ & 16 & $\mathrm{Nd}$ & $\mathrm{Nd}$ & 8 & $\mathrm{Nd}$ \\
\hline Microdesmis haumaniana & $\mathrm{Nd}$ & $\mathrm{Nd}$ & 32.1 & $\mathrm{Nd}$ & $\mathrm{Nd}$ & $\mathrm{Nd}$ & $\mathrm{Nd}$ & $\mathrm{Nd}$ \\
\hline Eriosema glomerata & 16 & $\mathrm{Nd}$ & $\mathrm{Nd}$ & 16.03 & $\mathrm{Nd}$ & 32 & $\mathrm{Nd}$ & 16 \\
\hline Haumania sp & $\mathrm{Nd}$ & $\mathrm{Nd}$ & $\mathrm{Nd}$ & $\mathrm{Nd}$ & $\mathrm{Nd}$ & $\mathrm{Nd}$ & $\mathrm{Nd}$ & $\mathrm{Nd}$ \\
\hline
\end{tabular}

\section{Discussion:-}

1. The nutritional value of a vegetable depends on its nutrient and anti-nutritional constituents (Aletor et al., 1994). The results of nutritional content analysis have shown that crude protein content was relatively higher in wild food plants from mandrill diet and used also by native population. It was highest in the seeds of Ricinodendron heudelotii, Pentaclethra macrophylla, and abundant in the fruits of Uapaca guineensis and Aframomum alboviolaceum. The protein content in those wild vegetables were comparable with or higher that crud protein content reported by some authors on exotic vegetables like lettuce, cabbage and spinach (Yildirim et al., 2001) commonly consumed in South Africa.

2. Our study shows also that crud protein content of twenty-two wild vegetables analyzed were ranged from $1.7 \pm$ $0.0 \%$ to $34.6 \pm 0.7 \%$ DM. Similar results have been also reported in Nigeria (Oguntona, 1998) and in Coruh Valley (Yildirim et al., 2001), where the crud protein contents of wild vegetables were observed to range between 3.5 and $32.5 \%$ DM. Whole results would suggest that some wild vegetables consumed by mandrills such as Ricinodendron heudelotii, Chrysophyllum africanum and Pentaclethra macrophylla would be a good protein sources.

3. Similarly, Pentaclethra macrophylla is considered as a good protein source and is used as protein supplement in Nigeria [Amata, 2010; Dike, 2010; Alinnor and Oze, 2011]. According to the institute of Medicine (RDA), the recommended dietary allowance of protein of children, men and women is 34, 56 and $46 \mathrm{~g} /$ day respectively (Satter et al., 2016). The mean of protein content in wild food plant from mandrill diet was of $12.55 \%$ dry matter and it is recommended that plant food provide more of $12 \%$ of calorie value from protein (Satter et al., 2016).

4. These observations show that some wild vegetables from mandrill diet would a good source of protein in human diet.

5. The carbohydrates are principal source of energy available. Our study has shown that carbohydrate content was ranged from $0.6 \pm 1.7$ to $78.3 \pm 2.1 \%$ DM. Some wild vegetables such as Chrysophyllum africanum and Chrysophyllum lacourtianum have shown higher carbohydrate content. These carbohydrate contents are higher or relatively higher of some wild edible leaves such as spinach and sweet potato which were $54.20 \%$ and $75.00 \%$ respectively (Imran et al., 2007).

6. However, these results on the other hand, were considerably higher than the values of $3 \%$ of wild edible plants of Pakistan and commonly consumed in Nigeria where the carbohydrate contents were ranged from 29.40 to $32.80 \%$ DM (Onwordi et al., 2009; Khan et al., 2013).

7. Thus, some wild food plants from mandrill diet would be good carbohydrates in native population diet.

8. The crud fiber of twenty-two food plants of mandrill was between $1.4 \pm 2.1$ and $41 \pm 1.0 \% \mathrm{DM}$ and the values the most higher were obtained with Rhynchospora corymbosa $(41 \pm 1.0 \% \mathrm{DM})$, Chrysophyllum africanum (21 $\pm 1.2 \% \mathrm{DM})$ and Megaphrynium macrostachum $(31 \pm 3.4 \% \mathrm{DM})$. These results are relatively high in relation to wild edible commonly consumed in Pakistan (Hussain et al., 2011; Shad et al., 2013).

9. The dietary fibers content necessary for digestion and promoting softs stools for effective elimination by adult males and females are 38 and 25 g/day respectively (Trumbo et al., 2002). It has been also shown that the consumption of vegetables relatively high in crude fibers to reduce serum cholesterol level, risk of coronary heart diseases and hypertension. It also increases glucose tolerance and insulin sensitivity (Araya et al., 2003). That suggests that some wild edible vegetables from mandrill diet like Chrysophyllum africanum would be a good source of dietary fibers in human diet.

10. Lipids are essential nutrients since they provide the body with maximum energy. Our study reveals that crude lipids content in wild vegetables such as Aframomum daniellii, Landolphia mannii and Pentaclethra macrophylla were very higher than $18.4 \%$ DM, reported for the leaves of Adansonia digitata (Osman, 2004).

11. Thus, crude lipids content of these vegetables used by mandrills can encourage their use in the human diet. 
12. Ash content in twenty-two plant species of our study is relatively highest especially for Aframomum daniellii, Landolphia mannii, Ricinodendron heudelotii and Palisota ambigua.

13. The caloric values of wild vegetables show that are highest in several species especially for Pentaclethra macrophylla and Ricinodendron heudelotii indicating that could be an important source of energy. Their caloric values are very highest than the values reported for Ocimum grattissimum $(408.70 \mathrm{Kcal} / 100 \mathrm{~g} \mathrm{DM})$ and Vernonia amygdalina $(406.13 \mathrm{Kcal} / 100 \mathrm{~g} \mathrm{DM})$ two wild edible from Nigeria (Udousoro and Ekanem, 2013). It has been reported that in healthy humans, the rate of gastric emptying is a function of caloric value (Calbet et al., 1997). On the whole, our results show that some wild edible vegetables from mandrill diet would be a good food source of energy and nutrient content usable in the human diet.

14. The content of ashes in leafy vegetables is a reflection of the amount of mineral elements present in the vegetables [Fagbohun et al., 2012].

15. The content of ashes of the plant food would imply the mineral content, of where very nourishing, but it has been reported that it would be the reverse if the ash contained toxic metals [Ukam, 2008].

16. Therefore, high ash content is not necessarily a conclusive factor regarding the health benefits of vegetables.

17. However, it is reported that with ash content greater than 8.8\% DM are healthful [Ifon and Bassir, 1980].

18. Thus, these food plants would be the good source of mineral content in the human diet.

19. The essential minerals ( $\mathrm{K}, \mathrm{Na}, \mathrm{Ca}, \mathrm{P}$ and $\mathrm{Mg}$ ) and trace minerals ( $\mathrm{Fe}, \mathrm{Cu}$ and $\mathrm{Zn}$ ) values of twenty-two wild food plants from mandrill diet show that the $\mathrm{Na}$ and $\mathrm{K}$ were the most abundant. Sodium and potassium are closely related in the body fluids. They regulate the acid-base balance. A diet high in potassium and low in sodium content has added advantage because of the direct relationship of sodium intake with hypertension in humans (Njoku and Akumefula, 2007).

20. According to Akinyeye et al. (2010), the recommended $\mathrm{Na} / \mathrm{K}$ ratio is 0.6 , and the values obtained with twentytwo vegetables analyzed was less than 0.6 except Palisota ambigua.

21. Thus, the wild food plants would be considered as good source of $\mathrm{K}$ and $\mathrm{Na}$ for effective health utilization.

22. The $\mathrm{Ca}$ values in the studied wild plants are higher except in Aframomum daniellii. These values were comparable with the values reported for wild green leafy vegetables used in North East India and for wild edible commonly consumed in Nigeria (Nkafamiya et al., 2010; Hussain et al., 2011; Saikia and Deka, 2013). High concentration of calcium in the body is very important because of its role in formation of bones and teeth, clotting of blood, muscle contraction and synaptic transmission of nerve impulses (Brody, 1994; Ghani et al., 2012). Calcium is an essential structural and functional element in living cells. It participates in cell division and the regulation of cell proliferation and differentiation (Whitfield et al., 1979). The $\mathrm{Ca}$ is an important macronutrient for the growth and maintenance of teeth, bone, muscle and heart function (Akubugwo et al., 2007). It is with phosphorus content was also low $(5.72 \mathrm{mg} / 100 \mathrm{~g})$. It is with phosphorus the minerals present in the largest quantity in the structure of the body and bones (Shills and Young, 1988). The phosphorus values found by wild vegetables were significantly comparable with the values reported in vegetables consumed in Western Côte d'Ivoire (Zoro et al., 2014). These data indicating that the wild vegetables from mandrill diet would provide a good source of minerals in human diets.

23. Our study reveals that the Fe values of some wild vegetables such as Aframomum cf polyanthum, Landolphia mannii and Medinilla mirabilis were very highest than the average values recommended in dietary intake which are of $400 \mathrm{~g} /$ day of fresh leafy vegetables that would result in an intake of about $40 \mathrm{~g}$ of dry vegetables per day [FAO, 2004]. The recommendation is an agreement with the results obtained in our study and proved that wild vegetables from mandrill diet would be a good source of $\mathrm{Fe}$ in human diet. Fe is important in the diet for the formation of hemoglobin, normal functioning of the central nervous system and in the metabolism of carbohydrates, proteins and fats (Kaya and Incekara, 2000; Gupta, 2014). It is also an important mineral in immune function, cognitive development, temperature regulation and energy metabolism (Wardlaw et al., 2004).

24. The Mg was relatively abundant in some plant like Aframomum alboviolaceum and Pentaclethra macrophylla. It is required in over 300 enzymes that utilize adenosine triphosphate. It contributes to DNA and RNA synthesis during cell proliferation. It is necessary for nerve and heart function as release of insulin and ultimate insulin action on cell and decreases also blood pressure by dilating arteries (Wardlaw et al., 2004).

25. The results of mineral composition have also shown that the $\mathrm{Zn}$ and $\mathrm{Cu}$ values recorded in different vegetables were higher. These values were significantly higher than those reported for wild vegetables consumed in Bangladesh (Satter et al., 2016). The $\mathrm{Zn}$ is important mineral which is required in antioxidant system of organism. It is the component anti-oxidative enzymes, superoxide dismutase, catalase and glutathione peroxidase. 
26. Also, $\mathrm{Zn}$ is required for the function of 200 enzymes and is important in growth and sexual development in man (Kawade, 2012).

27. Copper is important in diet since it is a very powerful pro-oxidant and catalyzes the oxidation of unsaturated fats and oil as well as ascorbic acid (John, 1999). The copper content in all studied will vegetables were highest compared to recommended in human diets since the RDA value for copper is $1.5-3 \mathrm{mg}$ for a male adult (NRC, 1989).

28. The phytochemical analysis results have revealed abundance in secondary compounds in food plants from mandrill diet and the highest content polyphenol compounds. The abundance in secondary metabolites in food plants consumed by Mandrillus sphinx supposes that the antinutritional compounds will be among some factors which may determine the selection of some plants in the mandrill as others primate species. Regarding the highest total phenols content of some plant species such as Aframomum cf polyanthum, Aframomum alboviolaceum and Pentaclethra macrophylla comparing with values reported by Amarowicz et al. (2004) on some medicinal plants reinforcing this hypothesis.

29. The use of secondary metabolites by animals especially by the primates has been reported in several studies. For example, chimpanzees are known to consume some plants with low nutritional value and/or high bioactive compounds possibly to maintain and improve their health suggesting a self-medicating role (Wrangham et al., 1983; Huffman and Seifu, 1989; Huffman and Wrangham, 1994; Kreif et al., 2005; Krief et al., 2008).

30. Antioxidant activities results of some food plants from mandrill diet and consumed by local people have demonstrated the high potential antioxidant activity index of fruits of Aframomum alboviolaceum (4.16 \pm 0.2$)$, Aframomum of polyanthum (5.19 \pm 3 ) and Pseudospondias longifolia (4.02 \pm 2.5$)$. These results in additional phytochemical results demonstrated that some wild plants from mandrill diet such as Aframomum alboviolaceum, Aframomum cf polyanthum and Pseudospondias longifolia, will be the good source of natural antioxidant. These wild vegetables may constitute an alternative in to prevent metabolic affections.

31. Indeed, it is established that flavonoids will be the good natural antioxidants (Cai et al., 2004). They inhibited the production of hypochlorite acid $(\mathrm{HOCl})$ by the human body (Pincemail et al., 2002).

32. Regarding antimicrobial activity of some plants from Mandrill diet, the results revealed that some plants tested present inhibitory activity on the majority bacterial strains isolated in the mandrill individual from the study population. This inhibitory activity observed will justify by abundant in antibacterial secondary metabolites such as tannins, alkaloids, flavonoids, saponins present in these food plants. It has been reported by several authors that many compounds as alkaloids, les tannins, les flavonoids and triterpenoids present the important potential antibacterial activity (Moroh et al., 2008; Bruneton, 2009; Tene et al., 2009).

33. Analyzing the zone inhibition diameters, the results attested that the majority of plants tested have showed the important inhibitory diameters recorded between 7 and $22 \mathrm{~mm}$.

34. In additional, some plant species as Medelina mirabilis, Pentaclethra eetveldeana, Piptadeniastrum africanum and Tristemma mauritianum inhibited the growth of bacterial strains (ECC110, EC121, EC292 and EC129) resistant at standard antibiotic (Ertapenem) used.

35. Our result revealed inhibition effect on bacterial isolates from mandrill of some plants attesting the bio-efficacy of certain plants consumed by this mandrill population. Indeed, it demonstrated that plant extracts are active, when their zone inhibitory diameter is $\geq 10 \mathrm{~mm}$ (Biyiti et al., 2004). The hypothesis of the efficacy of food plants from mandrills on bacterial strains isolates is reinforcing by the fact that, some plants also consumed have been study by others authors and the results obtained demonstrated antibacterial activity (N'guessan et al., 2007 ; Tekwu et al., 2012; Sima-Obiang et al., 2016a; 2016b). For example, fruits of Thonningia sanguine, Megaphrynium macrostachum, Xylopia aethiopica and Piper guineensis consumed by the study mandrill population present antimicrobial activity on multi-resistant isolates of Escherichia coli et Klebsiella pneumoniae producing the ß-lactamase enzymes, Mycobacterium tuberculosis, Pseudomonas aeruginosa ATCC 19249; Escherichia coli ATCC 0157, Enterococcus faecalis CIP 10907, Pseudomonas aeruginosa ATCC 19249, Escherichia coli ATCC 0157, Enterococcus faecalis CIP 10907, Pseudomonas aeruginosa ATCC 19249 (N'guessan et al., 2007 ; Tekwu et al., 2012; Sima-Obiang et al., 2016b).

36. Observation results of MIC and $\mathrm{MBC}$ confirmed antibacterial activity of food plants from mandrill diet. Analyzing the activity reports (MBC/MIC) of plant extracts, the results show that is ranged between 2 and 64 . It is demonstrated that if this activity report of extract is inferior at $4(\leq 4)$, it is considerate as presenting a bactericide activity and if it is superior at $4(\geq 4)$, the extract is considerate as bacteriostatic (Marmonier, 1990). Among plant species analyzed, T. mauritianum (Melastomataceae), M. schweinfurthii (Euphorbiaceae), M. mirabilis (Melastomataceae), A. floribunda (Euphorbiaceae) et P. eetveldeana (Mimosaceae) will have bactericide activity on bacterial strains (EC114, PA383 et EC292) and others will be bacteriostatic effect. 
37. This study demonstrate that among the diversity of plants consumed by Mandrillus sphinx, some such as Ricinodendron heudelotii, Pentaclethra macrophylla, Chrysophyllum africanum and Aframomum alboviolaceum will constitute an important source in calorie and mineral content. Others as Aframomum alboviolaceum, A. cf polyanthum and Pseudospondias longifolia present the good antioxidant activity.

38. These plants will be recommended in Human nutrition and can constitute an alternative against metabolic affections.

39. Concerning antibacterial activity, T. mauritianum, M. schweinfurthii, M. mirabilis, A. floribunda and $P$. eetveldeana will be the bactericide effect.

40. Thus, this study has permeated of showed that the mandrill will use their vegetable resources of their ecosystem for to respond at their need in energy, mineral and probably for their needs of heart.

41. In additional, the highest phenol compounds values in the plant parts from mandrill diet will permitted also of supposed that the use of important diversity of vegetables by this primate species (Hoshino, 1985; Lahm, 1986; Norris, 1988; Nsi Akoué et al., 2017; Nsi Akoué, 2017) will be justified by their needs in secondary metabolites for to prevent or control the microbial affections.

\section{Conclusion:-}

In the present study, analytical investigations were carried out to ascertain the chemical composition, nutritional proximate, antioxidant and antibacterial potentials of wild vegetables from mandrill diet.

The results showed that plant species consumed by this non-human primate species present an important of good nutritional sources of nutrients and mineral compounds. Also, that some plants will be of good natural antioxidants sources usable by local population. Among all plants analyzed, T. mauritianum, M. schweinfurthii, M. mirabilis, A. floribunda and P. eetveldeana present the bactericide effects and certain among other have bacteriostatic effects on gastrointestinal bacteria. Higher phenolic contents and others secondary metabolites in the alimentation of Mandrillus sphinx supposed that some food plants will be selected for their secondary metabolite contents and will explicate also the bactericide activity observed. These pharmacological results attested the bioefficacy of certain food plants consumed by this primate species.

Thus, insertion of certain of these vegetables in human nutrition is recommendable. These interesting primarily results will be completed by other analysis for a good knowledge of nutritional behavior of this primate species.

\section{Acknowledgment:-}

We thank the laboratory "CNRS-DPZ Mandrillus Project" and the staff of "Project Mandrillus" of Bakoumba, the Society of Exploitation of Lékédi Park (SODEPAL) and University of Sciences and Technique of Masuku (USTM) from Gabon for their contribution the realization of this work. We also thank the authority for have facility the realization of the survey and people for their availability and their implication in the realization of this study.

\section{References:-}

1. Akinyeye, R.O., Oluwadunsi, A. Omeyeni, A., 2010. Proximate, mineral, anti-nutrients, phytochemical screening and amino acid compositions of the leaves of Pterocarpus mildbraedi karms. Electron J. Environ. Agric. Food Chem. 94, 1322-1333.

2. Akubugwo, I.E., Obasi, N.A., Chinyere, G.C., Ugbogu, A.E., 2007. Nutritional and chemical value of Amaranthus hybridus L. leaves from Afikpo, Nigeria. Afr J Biotechnol. 6, 2833-2839.

3. Alinnor, I.J., Oze, R., 2011. Chemical evaluation of the nutritive value of Pentaclethra macrophylla benth (African Oil Bean) Seeds. Pak. J. Nutr. 10, 355-359.

4. Aletor, V.A., Goodchild, A.V., Abde, E.1., Moneim, A.M., 1994.Nutritional and anti-nutritional characteristics of selected vicia genotypes. Animal Feeding Sci. Technol. 47, 125-139.

5. Altmann, J., 1974. Observational study of behaviour: Sampling methods. Behaviour. 49, 227-267.

6. Amarowicz, R., Pink, D., Shahidi, F., 2004.Multivariate model for the prediction of soluble condensed tannins in crude extracts of polyphenols. J. Am. Oil Chem. Society-Springer. 5, 1-17.

7. Amata, I.A., 2010. Nutritional value of the leaves of Myrianthus arboreus: A Browse plant. Int. J. Agric. Res. 5, 576-581.

8. Araya, H., Pak, N., Vera, G., Alviña, M., 2003. Digestion rate of legume carbohydrates and glycaemic index of legume-based meals. Int. J. Food Sci. Nutr. 54, 119-126. 
9. Arvouet-Grand, A., Vennat, B., Pourrat, A., Legret, P., 1994.Standardization of propolis extract and identification of principal constituents. J. pharm. Belg. 49, 462.

10. Association of Official Analytical Chemists (AOAC). 1990. Official Methods of Analysis. 15th Edition, Association of Official Analytical Chemists, Washington DC.

11. Association of Official Analytical Chemist (AOAC). 1995. Official Methods of Analysis (15th Ed.), Washington D.C, pp. 600-792.

12. Association of Official Analytical Chemists (AOAC). 2000. Official Method of Analysis, 15th ed.; AOAC: Gaithersburg, MD, USA (No. 967.21 Ascorbic acid in vitamin preparations and juices).

13. Bidie, A.d.P., Banga, B., N'Guessan, Adou, F., Yapo, N'Guessan, J.D., Djaman, A.J., 2011. Activités antioxydantes de dix plantes médicinales de la pharmacopée ivoirienne. Sciences \& Nature. 8, 1-11.

14. Biyiti, L.F., Meko'o, D.J.L., Tamzc, v., Amvam Zollo, P.H., 2004.Recherche de l'activité antibactérienne de quatre plantes médicinales camerounaise. Pharm. Méd. Trad. Afr. 13, 11-20.

15. Bradford, M.A., 1976. Rapid and sensitive method for the quantization of microgram quantities of protein utilizing the principle of protein-dye binding. Anal. Biochem. 72, 248-254.

16. Brockmeyer, T., Kappeler, P.M., Willaume, E., Benoit, L., Mboumba, S., Charpentier, M.J.E., 2015. Social organization and space use of a wild mandrill (Mandrillus sphinx) group. Am. J. Primatol. 77, 1036-1048.

17. Brody, T., 1994.Nutritional Biochemistry. San Diego, CA, Academic Press. pp. 658.

18. Bruneton, J., 1999. Pharmacognosie, phytochimie, plantes médicinales. Technique et documentation. Lavoisier, Paris. pp. 458-953.

19. Bruneton, J., 2009. Pharmacognosie, Phytochimie, Plantes médicinales. Technique \& Documentation, Paris, pp. 721-741.

20. Bssaibis, F., Gmira, N., Meziane, M., 2009. Activité antibactérienne de Dittrichia viscoa (L.) W. Greuter. Rev. Microb. Ind. San. Environ. 3, 44-45.

21. Burhingame, B., 2000. Comparison of total lipids, Fatty acids, Sugars and Non Volatile Organic Acids in Nuts from Castanea species. J. Food Comp. Anal. 13, 99-100.

22. Cai, Y., Luo, Q., Sun, M., Corke, H., 2004. Antioxidant activity and phenolic compounds of 112 Chinese medicinal plants associated with anticancer. Life Sciences. 74, 2157-2184.

23. Calbet, J.A.L., MacLean, D.A., 1997. Role of caloric contents on gastric emptying in humans. J. Physiol. 498, 553-559.

24. Dike, M.C., 2010. Proximate, phytochemical and nutrient compositions of some fruits, seeds and leaves of some plant species at Umudike, Nigeria. J. Agric. Biol. Sci. 5, 7-16.

25. Dubois, M., Gilles, K.A., Hamilton, J.K., Rebers, P.A., Smith, F., 1956.Colorimetric method for determination of sugars and related substances. Anal. Chem. 28, 350-356.

26. Fasuyi, A.O., 2006. Nutritional potentials of some tropical vegetable meals. Chemical characterization and functional properties. Afr. J. Biotechnol. 5, 49-53.

27. Fernanda, B.M., polydoro, M.E., Andrades, M., Luiz, F., Dial-Pizzol., 2006. Effect of maternal protein malnutrition on oxidative markers in the young rat cortex and cerebellum. Neurosci. Lett. 406, 281-284.

28. Fagbohun, E.D., Lawal, O.U., Ore, M.E., 2012. The proximate, mineral and phytochemical analysis of the leaves of Ocimum grattissimum L., Melantherascandens A. and Leea guineensis L. and their medicinal value. Int. J. Appl. Biol. Pharm. Technol. 3, 15-22.

29. Food and Agriculture Organization/ World Health Organization (FAO). 2004. Fruits and vegetables for health: report of a joint FAO/WHO workshop. Rome: Food and Agriculture Organization; Geneva: World Health Organization; [Online] Available from: http:// www.who.int/dietphysicalactivity/publications/fruit_vegetables_report. .

30. Ghani, A., Ali, Z., Ishtiaq, M., Maqbool, M., Parveen, S., 2012. Estimation of macro and micro nutrients in some important medicinal plants of Soon Valley, District Khu-shab, Pakistan. Afr. J. Biotechnol. 11, 1438614391.

31. Gupta, C.P., 2014. Role of iron (Fe) in body. J. Appl. Chem. 7, 38-46.

32. Hoshino, J., 1985. Feeding ecology of mandrills (Mandrillus sphinx) in Campo Animal Reserve, Cameroon. Primates. 26, 248-273

33. Huffman, M.A., Seifu, M., 1989. Observations on the illness and consumption of a possibly medicinal plant Vernonia amygdalina (Del.), by a wild chimpanzee in the Mahale Mountains National Park, Tanzania. Primates. 30, 51-63.

34. Huffman, M.A., Wrangham, R.W., 1994. Diversity of medicinal plants use by chimpanzees in the wild. In: Wrangham, R.W., McGrew, W.C., de Waal, F.B.M., Heltne, P.G., editors. Chimpanzee cultures. Mass: Harvard University Press. pp. 129-148. 
35. Hussain, J., Rehman, N., Khan, A.L., Hussain, H., Ahmed, A., Ali, L., 2011. Determination of macro and micronutrients and nutritional prospects of six vegetable species of Mardan, Pakistan. Pak J Bot. 43, $2829-2833$.

36. Ifon, E.T., Bassir, O., 1980. The nutritive value of some Nigerian leafy green vegetable: The distribution of protein, carbohydrate, crude fat, fiber and ash. Food Chem. 1, 231-235.

37. Imran, M., Talpur, F.N., Jan, M.I., Khan, A., Khan, I., 2007. Analysis of nutritional components of some wild edible plants. J Chem. Soc Pak; 29, 500-505.

38. John, M.M., 1999. Principles of Food Chemistry, 3rd edition, 209-223. Lintas, C. 1992. Nutritional Aspects of Fruits and vegetables consumption. Options medierraeenes. 19, 79-87.

39. Kawade, R., 2012. Zinc status and its association with the health of adolescents: a review of studies in India. Glob Heath Action. 5, 7353.

40. Kaya, I., Incekara, N., 2000. Contents of some wild plants species consumed as food in Aegean region. J. Turk of Weed Sci. 3, 5664.

41. Khan, N., Sultana, A., Tahir, N., Jamila, N., 2013. Nutritional composition, vitamins, minerals and toxic heavy metals analysis of Trianthema portulacastrum L., a wild edible plant from Peshawar, Khyber Pakhtunkhwa, Pak. Afr J Biotechnol. 12, 6079-6085.

42. Keatinge, D., 2012.Vegetables: Less visible, but vital for human health. Why nutrient-dense indigenous vegetables must be on the plate for economic development, food security, and health. AVRDC News Brief.

43. Koudou, J., Obame, L.C., Edou, P., Bassolé, I., Eba, F., Figueredo, G., Traore, A.S., Chalchat, J.C., 2008. Volatile constituents, antioxidant and antibacterial properties of Daniella Klainei Pierre ex A. Chev. Essential oil. Sci. Res. Essays. 3, 316-319.

44. Krief, S., Thoison, O., Sévenet, T., Wrangham, R.W., Lavaud, C., 2005. Novel triterpenoid saponins isolated from the leaves of Albizia grandibracteata ingested by primates in Uganda. J. Nat. Prod. 68, 897-903.

45. Krief, S., Jamart, A., Mahé, S., Leendertz, F.H., Mätz-Rensing, K., Crespeau, F., Bain, O., Guillot, J., 2008. Clinical and pathological manifestation of oesophagostomosis in African great apes. Does self-medication in wild apes influence disease progression? J. Med. Primatol. 37, 188-195.

46. Kyung-Hee, K., Rong, T., Yang, R., Steve, W.C., 2005. Phenolic acid profiles and antioxidant activities of wheat bran extracts and the effect of hydrolysis conditions. Food Chem. 95, 466-473.

47. Lachman, J., Orsák, M., Pivec, V., 2000. Antioxidant contents and composition in some fruits and their role in human nutrition. Hortic. Sci. 27, 103-117.

48. Lahm, S.A., 1986. Diet and habitat preference of Mandrillus sphinx in Gabon: Implications of Foraging Strategy. Am. J. Primatol., 11:9-26.

49. Lennette, E.H., Truant, J.P., Spaulding, C.H., Abella, C., 1981. Manual de microbiología clínica. Salvat Editores.

50. Marmonier, A.A., 1990. Introduction aux techniques d'étude des antibiotiques. In Bacteriology medicaltechnicusually. Doin, Paris-France. pp 227-236.

51. Mohammed, A., Dangoggo S.M., Tsafe, A.I., Itodo, A.U., Atiku, F.A., 2011.Proximate, minerals antinutritional factors of Gardenia aqualla (Gauden duste) fruit pulp. Park. J. Nutr. 10, 577-581.

52. Moroh, J.L.A., Bahi, C., Dje, K., Loukouy, G., Guede-Guina, F., 2008. Étude de l'activité antibactérienne de l'extrait acétatique (EAC) de Morinda morindoides (Baker) milne-redheat (Rubiaceae) sur la croissance in-vitro des souches d'Escherichia coli. Bulletin de la Société Royale des Sciences de Liège. 77, 44-61.

53. N'guessan, J.D., Dinzedi, M.R., Guessennd, N., Coulibaly, A., Dosso, M.A.J., Djaman, A.J., Guede-Guina, F., 2007. Antibacterial activity of the aqueous extract of Thonningia sanguinea against Extended-Spectrum- $\beta$ Lactamases (ESBL) producing Escherichia coli and Klebsiella pneumoniae strains. Trop. J. Pharm. Res. 6, 779783.

54. Njoku, P.C., Akumefula, M.I., 2007.Phytochemical and nutrient evaluation of Spondias mombin leaves. Pak. J. Nutr. 6, 613-615.

55. Nkafamiya, I.I., Osemeahon, S.A., Modibbo, U.U., Aminu, A., 2010. Nutritional status of non-conventional leafy vegetables, Ficus asperifolia and Ficus sycomorus. Afr. J. Food Sci. 4, 104-108.

56. Norris, J., 1988. Diet and feeding behavior of semi-free ranging mandrills in an enclosed Gabonese forest. Primates. 29, 449-463.

57. Nsi Akoué, G., 2017. Mbading-Mbading, W.,Willaume E.,Souza A., Mbatchi B., Charpentier M.J.E., Seasonal and individual predictors of diet in a free-ranging population of mandrills. Ethology. 00, 1-14.

58. Nsi Akoué, G., 2017.Etude des plantes médicinales et/ou nutritionnelles issues du régime alimentaire du mandrill (Mandrillus sphinx) : Approche Zoopharmacologique. Thèse de doctorat unique de l'Université des Sciences et Techniques de Masuku (USTM) Franceville-Gabon. 
59. National Research Counci (NRC). 1989.Recommended Dietary allowances. National Academy Press, Washington, DC.

60. Obame, L.C., Koudou, J., Chalchat, J.C., Bassolé, I., Edou, P., Ouattara, A.S., Traore, A.S., 2007. Volatile components, antioxidant and antibacterial activities of Dacryodes buettneri HJ Lam. essential oil from Gabon. Sci. Res. Essays. 2, 491-495.

61. Onunogbu, I.C., 2002. Lipids in Human Existence, 1st ed.; AP Express Publishing Company: Nsukka, Nigeria.

62. Onwordi, C.T., Ogungbade, A.M., Wusu, A.D., 2009. The proximate and mineral composition of three leafy vegetables commonly consumed in Lagos, Nigeria. Afr J Pure Appl. Chem. 3, 102-107.

63. Osman, M.A., 2004. Chemical and nutrient analysis of baobab (Adansonia digitata) fruit and seed protein solubility. Plant foods human nutr. 59, 29-33.

64. Pearson, D., Egan, H., Kirk, R.S., Sawyer, R., 1981.Pearson's Chemical analysis of Food. Edinburgh: Churchill Livingston.

65. Peignot, P., Charpentier, M.J.E., Bout, N., Bourry, O., Massima, U., Dosimont, O., Terramorsi, R., Wickings, J., 2008. Learning from the first release project of captive-bred mandrills Mandrillus sphinx in Gabon. Oryx. 42, 122-131.

66. Pincemail, J., Bonjean, K., Cayeux, K., Defraigne, J.O., 2002. Physiological action of antioxidant defences. Nutrition Clinique et Métabolisme. 16, 233-239.

67. Poirotte, C., Basset, D., Willaume, E., Makaba, F., Kappeler, P.M., Charpentier, M.J.E., 2015. Environmental and Individual Determinants of parasite richness across seasons in a free-ranging population of mandrills (Mandrillus sphinx). Am. J. Phys. Anth., 159, 1-14.

68. Rodrigues, A.S., Perez-Gregorio, M.R., Garcia-Falcon M.S., Simal-Gandara, J., Almeida, D.P.F., 2010.Effect of post-harvest practices on flavonoid content of red and white onion cultivars. Food Control. 21, 878-884.

69. Saikia, P., Deka, D.C., 2013.Mineral content of some wild green leafy vegetables of North-East India. J. Chem. Pharm. Res. 5, 117-121.

70. Satter, M.M.A., Khan, M.M.R.L., Jabin, S.A., Abedin, N., Islam, M.F., Shaha, B., 2016. Nutritional quality and safety aspects of wild vegetables consumed in Bangladesh. Asian Pasific J. Trop. Biomed. 6, 125-131.

71. Scherer, R., Godoy, H.T., 2009. Antioxidant activity Index (AAI) by 2,2-diphenyl-1-picrylhydrazyl method. Food Chem. 112, 654-658.

72. Shad, A.A., Shah, H.U., Bakht, J., 2013. Ethnobotanical assessment and nutritive potential of wild food plants. J Anim Plant Sci. 23, 92-7.

73. Shills, M.E., G and Young, V.R., 1988. Nutrition, Brown publishers, Dubugye USA. pp 276-282.

74. Sima Obiang, C., Ondo J.P., Obame Engonga, L.C., Padzys, G.S., Zongo, C., Bongui, J.B., Nsi-Emvo, E., Traore, A., 2016a.Phytochemical screening, evaluation of antioxidant and antimicrobial properties of Erythrophleum ivorense A. Chev (Leguminosae) and Megaphrynium macrostachyum Benth (Marantaceae), medicinal plants from Gabon. Int. J. Biosci. 8, 43-53.

75. Sima Obiang, C., Ondo, J.P., Ndong Atome, G.R., Obame Engonga; L.C., Djoba Siawaya, J.F., Nsi Emvo, E., 2016b. Phytochemical screening, antioxidant and antimicrobial potential of stem barks of Cola edulis Baill. Pseudospondias longifolia Engl. and Carapa klaineana Pierre, from Gabon. Asian Pacific J. Trop. Disease, 6, 932-937.

76. Singleton, V.L., Orthofer, R., Lamuela-Raventos, R.M., 1999.Analysis of total phenols and other oxidation substrates and antioxidants by means of Folin-Ciocalteu reagent. Methods Enzymology. 299, 152-179.

77. Tene, M., Ndontsa, B.L., Tane, P., Tamokou, J.d.D., Kuiate, J.R., 2009. Antimicrobial diterpenoids and triterpenoids from the stem bark of Croton macrostachys. Int. J. Biol. Chem. Sci. 3, 538-544.

78. Tekwu, E.M., Tütin, A., Kuete, V., Nkengfack, A.E., Nyasse, B., Etao, F.X., Beng, V.P., 2012. Antimicrobial activity of selected Cameroonian dietary spices ethno-medically used against strains of Mycobacterium tuberculosis. J. Ethnopharm. 142, 374-382.

79. Trumbo, P., Schlicker, S., Yates, A.A., Poos, M., 2002. Food and Nutrition Board of the Institute of Medicine, the National Academies. Dietary reference intakes for energy, carbohydrates, fiber, fat, fatty acids, cholesterol, protein and amino acids. J Am Diet Assoc; 102, 1621-30.

80. Ukam, N.U. 2008. The potential of some lesser known vegetables. Nigerian J. Nutr. Sci. 29, 299-305.

81. Udousoro, I., Ekanem, P., 2013. Assessment of Proximate Compositions of Twelve Edible Vegetables in Nigeria. Int. J. Mod Chem, 4, 79-89.

82. Wada, L., Ou, B., 2002. Antioxidant activity and phenolic content of Oregon caneberries. J. Agric. Food Chem. 50, 3495-3500.

83. Wardlaw, G.M., Hampl, J.S., Disilvestro, R.A., 2004. Perspectives in nutrition. $6^{\text {th }}$ eds. New York: Mc Graw Hill. 
84. Whitfield, J.F., Boynton, A.L., Macmanus, J.P., Sikorska, M., Tsang, B.K., 1979. The regulation of cell proliferation by calcium and cyclic AMP. Mol. Cell. Biochemistry. 27, 155-159.

85. Wrangham, R.W., Nishida, T., 1983. Aspilia spp. leaves: a puzzle in the feeding behavior of wild chimpanzees. Primates. 24, 276-82.

86. Yildirim, E., Dursun, A., Turan, M., 2001. Determination of nutrition contents of the wild plants used as vegetables in upper Coruh Valley. Turkish J. Bot, 25, 367-371.

87. Zia-ul-haq, M., Iqbal, S., Ahmad, S., Bhanger, M.I., Wiczkowski, W., Amarowicz, R., 2008. Antioxidant potential of Desi chickpea varieties commonly consumed in Pakistan. J. Food Lipids.15, 326-342.

88. Zoro, A., Zoué, L., Megnanou, R.M., Koua, G., Niamké, S. 2014. Nutritive and antioxidant characteristics of roasted leafy vegetables consumed in Western Côte d'Ivoire (Ivory Coast). Am. J. Bio. Sci. 2, 196-202. 